\title{
Adaptive H(div)-Conforming Embedded-Hybridized Discontinuous Galerkin Finite Element Methods for the Stokes Problems
}

\author{
Yihui Han ${ }^{1}$ and Haitao Leng ${ }^{2, *}$ \\ 1 South China Research Center for Applied Mathematics and Interdisciplinary \\ Studies, South China Normal University, Guangzhou, 510630, Guangdong, China. \\ 2 School of Mathematical Sciences, South China Normal University, Guangzhou, \\ 510631, Guangdong, China.
}

Received 5 April 2021; Accepted 14 June 2021

\begin{abstract}
In this paper, we propose a residual-based a posteriori error estimator of embedded-hybridized discontinuous Galerkin finite element methods for the Stokes problems in two and three dimensions. The piecewise polynomials of degree $k(k \geq 1)$ and $k-1$ are used to approximate the velocity and pressure in the interior of elements, and the piecewise polynomials of degree $k$ are utilized to approximate the velocity and pressure on the inter-element boundaries. The attractive properties, named divergence-free and $\boldsymbol{H}$ (div)-conforming, are satisfied by the approximate velocity field. We prove that the a posteriori error estimator is robust in the sense that the ratio of the upper and lower bounds is independent of the mesh size and the viscosity. Finally, we provide several numerical examples to verify the theoretical results.
\end{abstract}

AMS subject classifications: 49M25, 65K10,65M50

Key words: Stokes equations, HDG methods, E-HDG methods, a posteriori error estimator, divergence-free, $\boldsymbol{H}$ (div)-conforming.

\section{Introduction}

In this paper, we consider the following Stokes problems: find the velocity $\boldsymbol{u}$ and pressure $p$ such that

$$
\begin{cases}-v \Delta \boldsymbol{u}+\nabla p=f, & \text { in } \Omega, \\ \nabla \cdot \boldsymbol{u}=\mathbf{0}, & \text { in } \Omega, \\ \boldsymbol{u}=\boldsymbol{g}, & \text { on } \partial \Omega .\end{cases}
$$

*Corresponding author. Email addresses: yhhan@m.scnu. edu.cn (Y. Han), htleng@m.scnu.edu.cn (H. Leng) 
Here $\Omega \subset \mathbb{R}^{d}(d=2,3)$ is an open, bounded, polygonal/polyhedral domain, $v>0$ is the dynamic viscosity and $f$ is the external body force. To keep compatibility, the boundary data $g$ needs to satisfy

$$
\int_{\partial \Omega} g \cdot \boldsymbol{n}=0,
$$

where $n$ is the unit vector normal to the boundary $\partial \Omega$.

Stokes problems are used to describe steady viscous incompressible flow, and the corresponding development of reliable and efficient a posteriori error analysis for finite element discretizations has been a very active field in recent decades, see $[7,8,29,31,32,45$, 46] and the references therein for more detail. We note that a framework for a posteriori error estimation for the Stokes problems has been provided in [29] based on the $\boldsymbol{H}^{1}$ conforming velocity reconstruction and the $\boldsymbol{H}$ (div)-conforming locally conservative flux (stress) reconstruction.

The hybridizable discontinuous Galerkin (HDG) method [14] provides a unifying strategy for hybridization of finite element methods for second order elliptic problems. By the local elimination of the unknowns defined in the interior of elements, the HDG method leads to a system where the unknowns are only the globally coupled degrees of freedom describing the introduced Lagrange multipliers. We refer to $[3,15,17,20,21$, $24,27,28,33,37,38,40,47]$ for some developments and applications for the HDG method. As for a posteriori error analysis of the HDG method, the authors in $[2,18,19]$ established a posteriori error estimates for second-order elliptic equations. For Stokes and Brinkman equations, the residual-based a posterior error estimator based on the gradientvelocity-pressure, velocity-pseudostress and velocity-pressure formulations were proved by $[3,23,30]$. Other related studies can be found in $[9,12,13,36]$.

The embedded discontinuous Galerkin (EDG) methods were proposed in $[16,26]$. The main difference between EDG and HDG methods is that the approximation spaces for Lagrange multipliers are continuous or not. Some developments of EDG methods could been found in $[11,22,35,39,48]$. On the other hand, we mention $[10,43]$ for an embeddedhybridized discontinuous Galerkin method for Stokes and Stokes-Darcy problems. It is worth noting that the approximate velocity field obtained by the embedded-hybridized discontinuous Galerkin method is $\boldsymbol{H}$ (div)-conforming and divergence-free. To the best of authors knowledge, there is no literature for a posteriori error analysis for this method.

In this paper, we develop a residual-based a posteriori error estimator for hybridized discontinuous Galerkin (HDG) and embedded-hybridized discontinuous Galerkin (EHDG) methods for the velocity-pressure formulation of Stokes problems in two and three dimensions. We have the following features:

- The piecewise polynomials of degree $k(k \geq 1)$ and $k-1$ are used to approximate the velocity and pressure in the interior of elements, and the piecewise polynomials of degree $k$ are adopted to approximate the velocity and pressure on the interelement boundaries. Certainly, the approximate velocity field is divergence-free and $\boldsymbol{H}$ (div)-conforming. 
- We introduce a posteriori error estimators for HDG and E-HDG methods, and we prove that the error estimators are reliable up to a data oscillation caused by the Dirichlet boundary condition. Moreover the efficiency of the error estimators are also obtained.

- Since the approximate space of numerical trace for velocity in E-HDG method is continuous, an extra error estimator (see (3.3)) needs to be introduced to ensure the reliability and efficiency.

The rest of the paper is organized as follows. Section 2 provides some notations and the HDG/E-HDG discrete schemes. We present a posteriori error estimator and discuss its reliability and efficiency in Section 3. We give the adaptive algorithm and confirm its results through several numerical examples in Section 4 . Finally, we conclude this paper with some conclusions in Section 5.

\section{Embedded-hybridized DG method}

\subsection{Notation and space}

For any open and bounded domain $D \subset \mathbb{R}^{d}(d=2,3)$, let $H^{m}(D)$ be the standard Hilbert spaces on the domain $D$ with the norm $\|\cdot\|_{m, D}$ and seminorm $|\cdot|_{m, D}$. If $m=0$, the space $H^{0}(D)$ will be abbreviated by $L^{2}(D)$ with the inner product $(\cdot, \cdot)_{D}$ for $D \subset \mathbb{R}^{d}$ or $\langle\cdot, \cdot\rangle_{D}$ for $D \subset \mathbb{R}^{d-1}$. When $D=\Omega$, we set $\|\cdot\|_{m}:=\|\cdot\|_{m, \Omega},|\cdot|_{m}:=|\cdot|_{m, \Omega}$, and $(\cdot, \cdot):=(\cdot, \cdot)_{\Omega}$. Furthermore, we define the following spaces:

$$
\begin{aligned}
& L_{0}^{2}(\Omega):=\left\{q \in L^{2}(\Omega):(q, 1)=0\right\}, \\
& \boldsymbol{H}(\operatorname{div}, D):=\left\{\boldsymbol{v} \in\left[L^{2}(D)\right]^{d}: \nabla \cdot \boldsymbol{v} \in L^{2}(D)\right\}, \\
& H_{0}^{1}(\Omega):=\left\{v \in H^{1}(\Omega): v=0 \text { on } \partial \Omega\right\} .
\end{aligned}
$$

Based on the domain $\Omega$, we consider a conforming and shape-regular partition $\mathcal{T}_{h}$. Let $\mathcal{T}_{h}^{\partial}$ denote the union of elements which have edge/face lying on the boundary of the domain $\Omega$. Denote $\varepsilon_{h}$ the set of all edges/faces of $\mathcal{T}_{h}, \varepsilon_{h}^{\partial}$ the set of all boundary edges/faces, and $\varepsilon_{h}^{i}$ the set of all interior edges/faces. We set $\partial \mathcal{T}_{h}:=\left\{\partial K: K \in \mathcal{T}_{h}\right\}$, where $\partial K$ is the set of all edges / faces of $K$. For any $K \in \mathcal{T}_{h}$ and $F \in \varepsilon_{h}, h_{K}$ and $h_{F}$ denote respectively the diameters of $K$ and $F$, and $\boldsymbol{n}_{K}$ denotes the outward unit normal vector on $\partial K$. Moreover, we denote by $h:=\max _{K \in \mathcal{T}_{h}} h_{K}$ the mesh size of $\mathcal{T}_{h}$, and by $\nabla_{h}$ and $\nabla_{h}$. the piecewise-defined gradient and divergence operators with respect to $\mathcal{T}_{h}$. To define the discrete scheme, we introduce the following mesh-dependent inner products and norms

$$
\begin{aligned}
&\langle u, v\rangle_{\partial \mathcal{T}_{h}}:=\sum_{K \in \mathcal{T}_{h}}\langle u, v\rangle_{\partial K}, \quad\|u\|_{0, \partial \mathcal{T}_{h}}:=\left(\sum_{K \in \mathcal{T}_{h}}\|u\|_{0, \partial K}^{2}\right)^{1 / 2}, \\
&(u, v)_{\mathcal{T}_{h}}:=\sum_{K \in \mathcal{T}_{h}}(u, v)_{K}, \quad\|u\|_{0, \mathcal{T}_{h}}:=\left(\sum_{K \in \mathcal{T}_{h}}\|u\|_{0, K}^{2}\right)^{1 / 2} .
\end{aligned}
$$


The jumps for tensor-valued function $w$ and vector-valued function $v$ and the average for $v$ on the interior edge/face $F \in \varepsilon_{h}^{i}$ are defined as

$$
\begin{aligned}
& \left.\llbracket \boldsymbol{w} \boldsymbol{n} \rrbracket\right|_{F}:=\left.(\boldsymbol{w} \boldsymbol{n})\right|_{K^{+} \cap F}+\left.(\boldsymbol{w} \boldsymbol{n})\right|_{K^{-} \cap F} \\
& \left.\llbracket \boldsymbol{v} \cdot \boldsymbol{n} \rrbracket\right|_{F}:=\left.(\boldsymbol{v} \cdot \boldsymbol{n})\right|_{K^{+} \cap F}+\left.(\boldsymbol{v} \cdot \boldsymbol{n})\right|_{K^{-} \cap F} \\
& \left.\{\boldsymbol{v}\}\right|_{F}:=\frac{1}{2}\left(\left.\boldsymbol{v}\right|_{K^{+} \cap F}+\left.\boldsymbol{v}\right|_{K^{-} \cap F}\right)
\end{aligned}
$$

where $K^{ \pm}$are two adjacent elements sharing a common face $F$.

Throughout the paper, we use $a \lesssim b(a \gtrsim b)$ to denote $a \leq C b(a \geq C b)$, where $C$ is a generic positive constant independent of mesh parameters $h, h_{K}, h_{F}$ and the viscosity coefficient $v$.

\subsection{The embedded-hybridized DG scheme}

Now we give the following finite element spaces:

$$
\begin{aligned}
& \boldsymbol{V}_{h}=\left\{\boldsymbol{v}_{h} \in\left(L^{2}(\Omega)\right)^{d}:\left.\boldsymbol{v}_{h}\right|_{K} \in\left(P_{k}(K)\right)^{d}, \forall K \in \mathcal{T}_{h}\right\}, \\
& Q_{h}=\left\{q_{h} \in L_{0}^{2}(\Omega),\left.q_{h}\right|_{K} \in P_{k-1}(K), \forall K \in \mathcal{T}_{h}\right\}, \\
& \overline{\boldsymbol{V}}_{h}=\left\{\boldsymbol{\mu}_{h} \in\left(L^{2}\left(\varepsilon_{h}\right)\right)^{d}:\left.\boldsymbol{\mu}_{h}\right|_{F} \in\left(P_{k}(F)\right)^{d}, \forall F \in \varepsilon_{h}\right\}, \\
& \overline{\boldsymbol{V}}_{h}^{g}=\left\{\boldsymbol{\mu} \in \overline{\boldsymbol{V}}_{h}:\left.\boldsymbol{\mu}\right|_{F}=\boldsymbol{I}_{h} \boldsymbol{g}, \forall F \in \varepsilon_{h}^{\partial}\right\}, \\
& \bar{Q}_{h}=\left\{\hat{q}_{h} \in L^{2}\left(\varepsilon_{h}\right):\left.\hat{q}_{h}\right|_{F} \in P_{k}(F), \forall F \in \varepsilon_{h}\right\},
\end{aligned}
$$

and

$$
\hat{V}_{h}^{g} \times \hat{Q}_{h}:= \begin{cases}\bar{V}_{h}^{g} \times \bar{Q}_{h}, & \text { HDG method, } \\ \left(\bar{V}_{h}^{g} \cap C^{0}\left(\Gamma_{0}\right)\right) \times \bar{Q}_{h}, & \text { E-HDG method, } \\ \left(\bar{V}_{h}^{g} \cap C^{0}\left(\Gamma_{0}\right)\right) \times\left(\bar{Q}_{h} \cap C^{0}\left(\Gamma_{0}\right)\right), & \text { EDG method, }\end{cases}
$$

where $P_{s}(D)$ denotes the set of polynomials of degree no larger than $s$ on the domain $D$, $\Gamma_{0}$ is the union of all edges/faces in $\varepsilon_{h}, \boldsymbol{I}_{h}$ is $L^{2}$-projection onto $\bar{V}_{h}$ for HDG method or a continuous interpolation for E-HDG and EDG methods.

Next, we define a bilinear form as follows:

$$
\begin{aligned}
\mathcal{B}\left(\boldsymbol{u}_{h}, \hat{\boldsymbol{u}}_{h}, p_{h}, \hat{p}_{h} ; \boldsymbol{v}_{h}, \boldsymbol{\mu}_{h}, q_{h}, \hat{q}_{h}\right):= & a_{h}\left(\left(\boldsymbol{u}_{h}, \hat{\boldsymbol{u}}_{h}\right),\left(\boldsymbol{v}_{h}, \boldsymbol{\mu}_{h}\right)\right)+b_{h}\left(\left(p_{h}, \hat{p}_{h}\right),\left(\boldsymbol{v}_{h}, \boldsymbol{\mu}_{h}\right)\right) \\
& -b_{h}\left(\left(q_{h}, \hat{q}_{h}\right),\left(\boldsymbol{u}_{h}, \hat{\boldsymbol{u}}_{h}\right)\right),
\end{aligned}
$$

where

$$
\begin{aligned}
a_{h}\left(\left(\boldsymbol{u}_{h}, \hat{\boldsymbol{u}}_{h}\right),\left(\boldsymbol{v}_{h}, \boldsymbol{\mu}_{h}\right)\right):= & \left(v \nabla_{h} \boldsymbol{u}_{h}, \nabla_{h} \boldsymbol{v}_{h}\right)_{\mathcal{T}_{h}}+\left\langle\tau\left(\boldsymbol{u}_{h}-\hat{\boldsymbol{u}}_{h}\right), \boldsymbol{v}_{h}-\boldsymbol{\mu}_{h}\right\rangle_{\partial \mathcal{T}_{h}} \\
& -\left\langle\boldsymbol{u}_{h}-\hat{\boldsymbol{u}}_{h}, v\left(\nabla_{h} \boldsymbol{v}_{h}\right) \boldsymbol{n}\right\rangle_{\partial \mathcal{T}_{h}}-\left\langle v\left(\nabla_{h} \boldsymbol{u}_{h}\right) \boldsymbol{n}, \boldsymbol{v}_{h}-\boldsymbol{\mu}_{h}\right\rangle_{\partial \mathcal{T}_{h}}, \\
b_{h}\left(\left(p_{h}, \hat{p}_{h}\right),\left(\boldsymbol{v}_{h}, \boldsymbol{\mu}_{h}\right)\right):= & -\left(p_{h}, \nabla_{h} \cdot \boldsymbol{v}_{h}\right)_{\mathcal{T}_{h}}+\left\langle\hat{p}_{h} \boldsymbol{n}, \boldsymbol{v}_{h}-\boldsymbol{\mu}_{h}\right\rangle_{\partial \mathcal{T}_{h} .} .
\end{aligned}
$$


Then the discrete scheme of problem (1.1) seeks $\left(\boldsymbol{u}_{h}, \hat{\boldsymbol{u}}_{h}, p_{h}, \hat{p}_{h}\right) \in \boldsymbol{V}_{\boldsymbol{h}} \times \hat{\boldsymbol{V}}_{h}^{g} \times Q_{h} \times \hat{Q}_{h}$ such that

$$
\mathcal{B}\left(\boldsymbol{u}_{h}, \hat{\boldsymbol{u}}_{h}, p_{h}, \hat{p}_{h} ; \boldsymbol{v}_{h}, \boldsymbol{\mu}_{h}, q_{h}, \hat{q}_{h}\right)=\left(\boldsymbol{f}, \boldsymbol{v}_{h}\right)_{\mathcal{T}_{h}}
$$

for all $\left(\boldsymbol{v}_{h}, \boldsymbol{\mu}_{h}, q_{h}, \hat{q}_{h}\right) \in \boldsymbol{V}_{\boldsymbol{h}} \times \hat{\boldsymbol{V}}_{h}^{0} \times Q_{h} \times \hat{Q}_{h}$. Here the stabilization function $\tau$ is defined by

$$
\tau=v \alpha_{v} h_{K}^{-1} \text { on } \partial K \text { with } K \in \mathcal{T}_{h}
$$

where the penalty parameter $\alpha_{v}>\alpha_{0}>0$ ( $\alpha_{0}$ is a positive constant defined in [41, Lemma 4.2] and [43, Lemma 2]). In implementation, the penalty parameter is taken as $\alpha_{v}=6 k^{2}$ for HDG method, and as $\alpha_{v}=4 k^{2}$ for E-HDG and EDG methods.

Remark 2.1. For the discrete scheme (2.5), the following properties hold:

- Consistency, i.e.

$$
\mathcal{B}\left(\boldsymbol{u}-\boldsymbol{u}_{h}, \boldsymbol{u}-\hat{\boldsymbol{u}}_{h}, p-p_{h}, p-\hat{p}_{h} ; \boldsymbol{v}_{h}, \boldsymbol{u}_{h}, q_{h}, \hat{q}_{h}\right)=0,
$$

for all $\left(\boldsymbol{v}_{h}, \boldsymbol{\mu}_{h}, q_{h}, \hat{q}_{h}\right) \in \boldsymbol{V}_{\boldsymbol{h}} \times \hat{\boldsymbol{V}}_{h}^{\mathbf{0}} \times Q_{h} \times \hat{Q}_{h}$.

- By taking $\left(\boldsymbol{v}_{h}, \boldsymbol{\mu}_{h}, q_{h}, \hat{q}_{h}\right)=\left(\mathbf{0}, \mathbf{0}, \nabla_{h} \cdot \boldsymbol{u}_{h}, 0\right)$ in (2.5), we can get

$$
\left\|\nabla_{h} \cdot \boldsymbol{u}_{h}\right\|_{\mathcal{T}_{h}}^{2}=0
$$

which indicates that the approximate velocity field is pointwise divergence-free within an element.

- For the HDG and E-HDG methods, the facet pressure $\widehat{p}_{h}$ is discontinuous. By taking $\left(\boldsymbol{v}_{h}, \boldsymbol{\mu}_{h}, q_{h}\right)=(\mathbf{0}, \mathbf{0}, 0)$ and

$$
\hat{q}_{h}= \begin{cases}\llbracket \boldsymbol{u}_{h} \cdot \boldsymbol{n} \rrbracket_{F}, & \text { on } F \in \varepsilon_{h^{\prime}}^{i} \\ \left(\boldsymbol{u}_{h}-\hat{\boldsymbol{u}}_{h}\right) \cdot \boldsymbol{n}, & \text { on } F \in \varepsilon_{h^{\prime}}^{\partial}\end{cases}
$$

in (2.5), we have

$$
\begin{array}{ll}
\llbracket \boldsymbol{u}_{h} \cdot \boldsymbol{n} \rrbracket_{F}=0, & \text { on } F \in \varepsilon_{h}^{i}, \\
\left(\boldsymbol{u}_{h}-\hat{\boldsymbol{u}}_{h}\right) \cdot \boldsymbol{n}_{F}=0, & \text { on } F \in \varepsilon_{h}^{\partial} .
\end{array}
$$

This shows that the discrete scheme (2.5) is $\boldsymbol{H}(\mathrm{div})$-conforming for HDG and EHDG methods.

- By taking $\left(\boldsymbol{v}_{h}, q_{h}, \hat{q}_{h}\right)=(\mathbf{0}, 0,0)$ in $(2.5)$, we have

$$
-\left\langle\tau\left(\boldsymbol{u}_{h}-\hat{\boldsymbol{u}}_{h}\right), \boldsymbol{\mu}_{h}\right\rangle_{\partial \mathcal{T}_{h}}+\left\langle v\left(\nabla_{h} \boldsymbol{u}_{h}\right) \boldsymbol{n}, \boldsymbol{\mu}_{h}\right\rangle_{\partial \mathcal{T}_{h}}=0, \quad \forall \boldsymbol{\mu}_{h} \in \hat{\boldsymbol{V}}_{h}^{0} .
$$

Then for the HDG method, we have from (2.11)

$$
\llbracket v\left(\nabla_{h} \boldsymbol{u}_{h}\right) \boldsymbol{n} \rrbracket-2\left\{\tau\left(\boldsymbol{u}_{h}-\hat{\boldsymbol{u}}_{h}\right)\right\}=0, \quad \text { on } F \in \varepsilon_{h}^{i} .
$$




\section{A Posteriori error estimation}

This section is devoted to a posteriori error analysis for the discrete scheme (2.5) for HDG method and E-HDG method with $\boldsymbol{g}=\mathbf{0}$. Moreover, in the following analysis, we need to assume that the pressure $\mathrm{p}$ is continuous across the interior face $F \in \varepsilon_{h}^{i}$.

Now we define a posteriori error estimator $\eta$ by

$$
\eta^{2}=\sum_{K \in \mathcal{T}_{h}}\left\{\eta_{K}^{2}+\eta_{\partial K}^{2}+\gamma \eta_{J}^{2}\right\}
$$

where

$$
\gamma= \begin{cases}0, & \text { HDG method, } \\ 1, & \text { E-HDG method, }\end{cases}
$$

and

$$
\begin{aligned}
& \eta_{K}^{2}=v^{-1} h_{K}^{2}\left\|\boldsymbol{f}+v \Delta \boldsymbol{u}_{h}-\nabla_{h} p_{h}\right\|_{0, K^{\prime}}^{2} \\
& \eta_{\partial K}^{2}=\left\|\tau^{\frac{1}{2}}\left(\boldsymbol{u}_{h}-\hat{\boldsymbol{u}}_{h}\right)\right\|_{0, \partial K^{\prime}}^{2} \\
& \eta_{J}^{2}=v^{-1} h_{K}\left\|\llbracket\left(v \nabla_{h} \boldsymbol{u}_{h}-p_{h} \boldsymbol{I}\right) \boldsymbol{n} \rrbracket\right\|_{0, \partial K \backslash \varepsilon_{h}^{\partial}}^{2} .
\end{aligned}
$$

Here $\boldsymbol{I}$ is the identity tensor and

$$
\|\llbracket \cdot \rrbracket\|_{s, \partial K \backslash \varepsilon_{h}^{\partial}}^{2}:=\sum_{F \in \partial K \backslash \varepsilon_{h}^{\partial}}\|\llbracket \cdot \rrbracket\|_{s, F}^{2} \cdot
$$

Next, we define the data oscillation corresponding to the source term $f$ by

$$
\begin{aligned}
& \operatorname{osc}\left(f ; \mathcal{T}_{h}\right)=\left(\sum_{K \in \mathcal{T}_{h}} \operatorname{osc}^{2}(f ; K)\right)^{\frac{1}{2}}, \\
& \operatorname{osc}(f ; K)=v^{-\frac{1}{2}} h_{K}\left\|f-\boldsymbol{P}_{h}^{k} f\right\|_{0, K^{\prime}}^{2}
\end{aligned}
$$

where $\boldsymbol{P}_{h}^{k}:\left(L^{2}(\Omega)\right)^{d} \rightarrow \boldsymbol{V}_{h}$ is the standard $L^{2}$-projection operator. For the nonhomogeneous Dirichlet boundary condition, we define

$$
\operatorname{osc}^{2}(\boldsymbol{g}, \partial \Omega)=\sum_{F \in \varepsilon_{h}^{\partial}} v\left(h_{F}^{-1}\left\|\boldsymbol{g}-\boldsymbol{I}_{h} \boldsymbol{g}\right\|_{0, F}^{2}+\left\|\boldsymbol{g}-\boldsymbol{I}_{h} \boldsymbol{g}\right\|_{\frac{1}{2}, F}^{2}\right) .
$$

Now we introduce some well-known results that will play an important role in the subsequent proofs. 
Lemma 3.1 ([42, Definition 1]). (BDM lifting operator) For any $\hat{q}_{h} \in R_{k}(\partial K):=\left\{\phi \in L^{2}(\partial K)\right.$ : $\left.\left.\phi\right|_{F} \in P_{k}(F), F \in \partial K\right\}$, there exists a BDM lifting operator $L^{B D M}: R_{k}(\partial K) \rightarrow\left(P_{k}(K)\right)^{d}$, which is a local lifting of the normal trace defined via BDM interpolant (c.f. [4, Example 2.5.1]) with zero on the interior, and has the properties:

$$
\left(L^{B D M} \hat{q}_{h}\right) \cdot \boldsymbol{n}=\hat{q}_{h} \quad \text { and } \quad\left\|L^{B D M} \hat{q}_{h}\right\|_{0, K} \lesssim h_{K}^{\frac{1}{2}}\left\|\hat{q}_{h}\right\|_{0, \partial K}, \quad \forall \hat{q}_{h} \in R_{k}(\partial K) .
$$

In addition, by an inverse estimate and the trace inequality, it also holds

$$
\begin{aligned}
& \left\|\nabla L^{B D M} \hat{q}_{h}\right\|_{0, K}^{2} \lesssim h_{K}^{-2}\left\|L^{B D M} \hat{q}_{h}\right\|_{0, K}^{2} \lesssim h_{K}^{-1}\left\|\hat{q}_{h}\right\|_{0, \partial K}^{2} \\
& \left\|L^{B D M} \hat{q}_{h}\right\|_{0, \partial K}^{2} \lesssim h_{K}^{-1}\left\|L^{B D M} \hat{q}_{h}\right\|_{0, K}^{2} \lesssim\left\|\hat{q}_{h}\right\|_{0, \partial K}^{2} .
\end{aligned}
$$

Lemma 3.2. For each element $K \in \mathcal{T}_{h}$ and any given nonnegative integer $j$, the following estimates hold

$$
\begin{array}{ll}
|v|_{1, K} \lesssim h_{K}^{-1}\|v\|_{0, K}, \quad \forall v \in \mathcal{P}_{j}(K), & \\
\|v\|_{0, \partial K} \lesssim h_{K}^{-1 / 2}\|v\|_{0, K}+h_{K}^{1 / 2}\|\nabla v\|_{0, K}, & \forall v \in H^{1}(K), \\
\|\boldsymbol{v} \cdot \boldsymbol{n}\|_{-1 / 2, \partial K} \lesssim\|\boldsymbol{v}\|_{0, K}+h_{K}\|\nabla \cdot \boldsymbol{v}\|_{0, K}, & \forall \boldsymbol{v} \in \boldsymbol{H}(\operatorname{div}, K) .
\end{array}
$$

Proof. The result (3.7) is the inverse estimate, which can be found in [44]. With trace theorem and the Cauchy-Schwarz inequality, we can obtain the approximation result (3.8). The last estimation (3.9) can be found in [25].

Lemma 3.3 ([6, Lemma 2.4]). Let $F$ be a face of the element $K \in \mathcal{T}_{h}, \boldsymbol{n}_{F}$ the unit vector normal to $F$, and $s>0$. Assume that $v$ is a given function in $H^{1+s}(K)$ and $\Delta v \in L^{2}(K)$. Then for any $w_{h} \in \mathcal{P}^{k}(F)$ we have

$$
\left\langle\nabla v \cdot \boldsymbol{n}_{F}, w_{h}\right\rangle_{F} \lesssim h_{F}^{-1 / 2}\left\|w_{h}\right\|_{0, F}\left(\|\nabla v\|_{0, K}+h_{K}\|\Delta v\|_{0, K}\right) .
$$

Following the [44], we give the definition of projection-mean operator. Given any point $a \in \Omega$, let $S_{h}^{c}(a):=\left\{K \mid K \in \mathcal{T}_{h}, a \in \bar{K}\right\}$ and $N_{h}^{c}(a)$ be the number of elements in $S_{h}^{c}(a)$. Then we define $\tilde{\boldsymbol{I}}_{h}:\left(L^{2}(\Omega)\right)^{d} \rightarrow \boldsymbol{V}_{h} \cap\left(H_{0}^{1}(\Omega)\right)^{d}$. For any $K \in \mathcal{T}_{h}, \boldsymbol{v} \in \boldsymbol{L}^{2}(\Omega),\left.\tilde{\boldsymbol{I}}_{h} \boldsymbol{v}\right|_{K} \in \boldsymbol{P}_{k}(K)$ satisfying

$$
\begin{aligned}
& \left.\tilde{\boldsymbol{I}}_{h} \boldsymbol{v}\right|_{K}\left(a_{i, K}\right)=\mathbf{0}, \quad \text { for any } a_{i, K} \in \partial \Omega, \\
& \left.\tilde{\boldsymbol{I}}_{h} \boldsymbol{v}\right|_{K}\left(a_{i, K}\right)=\left.\frac{1}{N_{h}^{c}\left(a_{i, K}\right)} \sum_{K^{\prime} \in S_{h}^{c}\left(a_{i, K}\right)}\left(\boldsymbol{P}_{h}^{k} \boldsymbol{v}\right)\right|_{K^{\prime}}\left(a_{i, K^{\prime}}\right), \quad \text { for any } a_{i, K} \text { is on } \partial K \text { and } a_{i, K} \notin \partial \Omega, \\
& \left.\tilde{\boldsymbol{I}}_{h} \boldsymbol{v}\right|_{K}\left(a_{i, K}\right)=\left.\boldsymbol{P}_{h}^{k} \boldsymbol{v}\right|_{K}\left(a_{i, K}\right), \quad \text { for any } a_{i, K} \text { is not on } \partial K \text { but in } K .
\end{aligned}
$$

Then the following error estimates hold. 
Lemma 3.4 ([44, Lemma 3.5.7]). Let $\tilde{\boldsymbol{I}}_{h}:\left(L^{2}(\Omega)\right)^{d} \rightarrow \boldsymbol{V}_{h} \cap\left(H_{0}^{1}(\Omega)\right)^{d}$ be the projection mean operator defined above. We have

$$
\left\|\boldsymbol{v}-\tilde{\boldsymbol{I}}_{h} \boldsymbol{v}\right\|_{0, K}+h_{K}^{\frac{1}{2}}\left\|\boldsymbol{v}-\tilde{\boldsymbol{I}}_{h} \boldsymbol{v}\right\|_{0, \partial K} \lesssim h_{K}\left(\sum_{K^{\prime} \in S_{h}(K)}\|\nabla \boldsymbol{v}\|_{0, K^{\prime}}\right)^{\frac{1}{2}}, \quad \forall \boldsymbol{v} \in\left(H_{0}^{1}(\Omega)\right)^{d},
$$

for any $K \in \mathcal{T}_{h}$. And $S_{h}(K):=\left\{K^{\prime}: K^{\prime} \in \mathcal{T}_{h}, \bar{K}^{\prime} \cap \bar{K} \neq \varnothing\right\}$.

Now we introduce the Oswald interpolation as: for $\left.\boldsymbol{g}_{D} \in \mathcal{C}(\Omega) \cap \bar{V}_{h}\right|_{\partial \Omega}$,

$$
\tilde{\boldsymbol{I}}_{h}^{o s}: \boldsymbol{V}_{h} \rightarrow \boldsymbol{V}_{h, g_{D}}^{c}:=\left\{\boldsymbol{v} \in \boldsymbol{C}(\Omega):\left.\boldsymbol{v}\right|_{K} \in \boldsymbol{P}_{k}(K), \forall K \in \mathcal{T}_{h},\left.\boldsymbol{v}\right|_{\partial \Omega}=\boldsymbol{g}_{D}\right\}
$$

Then we have the following results.

Lemma 3.5 ([34]). If the Dirichlet data $g_{D}$ is contained in $\left.C(\Omega) \cap \bar{V}_{h}\right|_{\partial \Omega}$, then for any $v_{h} \in V_{h}$ and any multi-index $\alpha$ with $|\alpha|=0,1$, the following estimate holds:

$$
\sum_{K \in \mathcal{T}_{h}}\left\|\boldsymbol{D}^{\alpha}\left(\boldsymbol{v}_{h}-\tilde{\boldsymbol{I}}_{h}^{o s} \boldsymbol{v}_{h}\right)\right\|_{0, K}^{2} \lesssim \sum_{F \in \varepsilon_{h}^{i}} h_{F}^{1-2|\alpha|}\left\|\llbracket \boldsymbol{v}_{h} \rrbracket\right\|_{0, F}^{2}+\sum_{F \in \varepsilon_{h}^{\partial}} h_{F}^{1-2|\alpha|}\left\|\boldsymbol{g}_{D}-\boldsymbol{v}_{h}\right\|_{0, F}^{2} \cdot
$$

Lemma $3.6([4,43])$. If the partition $\mathcal{T}_{h}$ consists of triangles in two dimensions or tetrahedra in three dimensions, there exists an interpolation operator $\boldsymbol{\Pi}_{\mathrm{BDM}}:\left(H^{1}(\Omega)\right)^{d} \rightarrow \boldsymbol{V}_{h}$ with the following properties for all $\boldsymbol{u} \in \boldsymbol{H}^{k+1}(K)$ where $k \geq 1$

- $\llbracket \boldsymbol{n} \cdot \Pi_{\mathrm{BDM}} \boldsymbol{u} \rrbracket=0$ on interior edges/faces.

- $\left\|\boldsymbol{u}-\boldsymbol{\Pi}_{\mathrm{BDM}} \boldsymbol{u}\right\|_{m, K} \leq c h_{K}^{l-m}|\boldsymbol{u}|_{l, K}$ with $m=0,1,2$ and $m \leq l \leq k+1$.

- $\left\|\nabla \cdot\left(\boldsymbol{u}-\boldsymbol{\Pi}_{\mathrm{BDM}} \boldsymbol{u}\right)\right\|_{m, K} \leq c h_{K}^{l-m}|\nabla \cdot \boldsymbol{u}|_{l, K}$ with $m=0,1$ and $m \leq l \leq k$.

- $\int_{K} q\left(\nabla \cdot \boldsymbol{u}-\nabla \cdot \boldsymbol{\Pi}_{\mathrm{BDM}} \boldsymbol{u}\right) \mathrm{d} x=0$ for all $q \in P_{k-1}(K)$.

- $\int_{F} \bar{q}\left(\boldsymbol{n} \cdot \boldsymbol{u}-\boldsymbol{n} \cdot \boldsymbol{\Pi}_{\mathrm{BDM}} \boldsymbol{u}\right) \mathrm{d} s=0$ for all $\bar{q} \in P_{k}(F)$, where $F$ is a face on $\partial K$.

\subsection{Reliability}

Now we are ready to prove the reliability of a posteriori error estimator $\eta$. Before this, we first provide a property in the following lemma between local error estimators.

Lemma 3.7. Let $\eta_{K}$ and $\eta_{\partial K}$ be defined in (3.1) and (3.2), then for each $K \in \mathcal{T}_{h}$, we have

$$
v^{-\frac{1}{2}} h_{K}^{\frac{1}{2}}\left\|p_{h}-\hat{p}_{h}\right\|_{0, \partial K} \lesssim \eta_{\partial K}+\eta_{K}
$$


Proof. By taking $\left(\boldsymbol{\mu}_{h}, q_{h}, \hat{q}_{h}\right)=(\mathbf{0}, 0,0)$ in the equation (2.5), we have for any $\boldsymbol{v}_{h} \in\left(P_{k}(K)\right)^{d}$

$$
\begin{aligned}
\left\langle\left(p_{h}-\hat{p}_{h}\right) \boldsymbol{n}, \boldsymbol{v}_{h}\right\rangle_{\partial K}= & -\left(\boldsymbol{f}+v \Delta \boldsymbol{u}_{h}-\nabla_{h} p_{h}, \boldsymbol{v}_{h}\right)_{K}+\left\langle\tau\left(\boldsymbol{u}_{h}-\hat{\boldsymbol{u}}_{h}\right), \boldsymbol{v}_{h}\right\rangle_{\partial K} \\
& -\left\langle\boldsymbol{u}_{h}-\hat{\boldsymbol{u}}_{h}, v\left(\nabla_{h} \boldsymbol{v}_{h}\right) \boldsymbol{n}\right\rangle_{\partial K} .
\end{aligned}
$$

Since $p_{h}-\hat{p}_{h} \in R_{k}(\partial K)$, from Lemma 3.1 we know that there exists a lifting operator $L^{B D M}$ such that $\left(L^{B D M}\left(p_{h}-\hat{p}_{h}\right)\right) \cdot \boldsymbol{n}=p_{h}-\hat{p}_{h}$. Then setting $\boldsymbol{v}_{h}=L^{B D M}\left(p_{h}-\hat{p}_{h}\right)$ in the above equality, we can get

$$
\begin{aligned}
& \left\|p_{h}-\hat{p}_{h}\right\|_{0, \partial K}^{2} \\
= & -\left(\boldsymbol{f}+v \Delta \boldsymbol{u}_{h}-\nabla_{h} p_{h}, \boldsymbol{v}_{h}\right)_{K}+\left\langle\tau\left(\boldsymbol{u}_{h}-\hat{\boldsymbol{u}}_{h}\right), \boldsymbol{v}_{h}\right\rangle_{\partial K} \\
& -\left\langle\boldsymbol{u}_{h}-\hat{\boldsymbol{u}}_{h}, v\left(\nabla_{h} \boldsymbol{v}_{h}\right) \boldsymbol{n}\right\rangle_{\partial K} \\
\lesssim & \eta_{K} v^{\frac{1}{2}} h_{K}^{-1}\left\|L^{B D M}\left(p_{h}-\hat{p}_{h}\right)\right\|_{0, K} \\
& +\eta_{\partial K} v^{\frac{1}{2}}\left(h_{K}^{-\frac{1}{2}}\left\|L^{B D M}\left(p_{h}-\hat{p}_{h}\right)\right\|_{0, \partial K}+h_{K}^{1 / 2}\left\|\nabla_{h}\left(L^{B D M}\left(p_{h}-\hat{p}_{h}\right)\right)\right\|_{0, \partial K}\right) \\
\lesssim & \left(\eta_{K}+\eta_{\partial K}\right) v^{\frac{1}{2}} h_{K}^{-\frac{1}{2}}\left\|p_{h}-\hat{p}_{h}\right\|_{0, \partial K},
\end{aligned}
$$

by (3.4)-(3.6) and (3.8). Then we conclude the proof.

Lemma 3.8. Let $(\boldsymbol{u}, p)$ and $\left(\boldsymbol{u}_{h}, \hat{\boldsymbol{u}}_{h}, p_{h}, \hat{p}_{h}\right)$ be the solutions of problems (1.1) and (2.5). We assume here that the pressure $p$ is continuous, and $g=0$ for E-HDG method. Then for any $\delta>0$, it holds

$$
\left\|v^{\frac{1}{2}}\left(\nabla \boldsymbol{u}-\nabla_{h} \boldsymbol{u}_{h}\right)\right\|_{0, \mathcal{T}_{h}}+\left\|\tau^{\frac{1}{2}}\left(\boldsymbol{u}_{h}-\hat{\boldsymbol{u}}_{h}\right)\right\|_{0, \partial \mathcal{T}_{h}} \lesssim \frac{1}{\delta}(\eta+o s c(g, \partial \Omega))+\delta\left\|v^{-\frac{1}{2}}\left(p-p_{h}\right)\right\|_{\mathcal{T}_{h}} \text {. }
$$

Proof. Firstly we set

$$
\begin{aligned}
& \delta_{u}=\boldsymbol{u}-\boldsymbol{u}_{h}-\boldsymbol{\Pi}_{\mathrm{BDM}}\left(\boldsymbol{u}-\boldsymbol{u}_{h}\right), \\
& \delta_{p}=p-p_{h}-P_{h}^{k-1}\left(p-p_{h}\right), \\
& \delta_{\hat{u}}=\boldsymbol{u}-\hat{\boldsymbol{u}}_{h}-\boldsymbol{\mu}_{h}, \quad \forall \boldsymbol{\mu}_{h} \in \hat{\boldsymbol{V}}_{h}^{0}, \\
& \delta_{\hat{p}}=p-\hat{p}_{h}-\hat{q}_{h}, \quad \forall \hat{q}_{h} \in \hat{Q}_{h},
\end{aligned}
$$

where $P_{h}^{s}$ is the scalar version of the operator $P_{h}^{s}$. Then with the definition of operator $\mathcal{B}$, we have

$$
\begin{aligned}
& \mathcal{B}\left(\boldsymbol{u}-\boldsymbol{u}_{h}, \boldsymbol{u}-\hat{\boldsymbol{u}}_{h}, p-p_{h}, p-\hat{p}_{h} ; \boldsymbol{u}-\boldsymbol{u}_{h}, \boldsymbol{u}-\hat{\boldsymbol{u}}_{h}, p-p_{h}, p-\hat{p}_{h}\right) \\
= & \left\|v^{\frac{1}{2}} \nabla_{h}\left(\boldsymbol{u}-\boldsymbol{u}_{h}\right)\right\|_{0, \mathcal{T}_{h}}^{2}+\left\|\tau^{\frac{1}{2}}\left(\boldsymbol{u}_{h}-\hat{\boldsymbol{u}}_{h}\right)\right\|_{0, \partial \mathcal{T}_{h}}^{2}-2\left\langle\hat{\boldsymbol{u}}_{h}-\boldsymbol{u}_{h}, v \nabla_{h}\left(\boldsymbol{u}-\boldsymbol{u}_{h}\right) \boldsymbol{n}\right\rangle_{\partial \mathcal{T}_{h}} .
\end{aligned}
$$

By integration by parts and (2.7), we yield

$$
\begin{aligned}
& \mathcal{B}\left(\boldsymbol{u}-\boldsymbol{u}_{h}, \boldsymbol{u}-\hat{\boldsymbol{u}}_{h}, p-p_{h}, p-\hat{p}_{h} ; \boldsymbol{u}-\boldsymbol{u}_{h}, \boldsymbol{u}-\hat{\boldsymbol{u}}_{h}, p-p_{h}, p-\hat{p}_{h}\right) \\
= & \mathcal{B}\left(\boldsymbol{u}-\boldsymbol{u}_{h}, \boldsymbol{u}-\hat{\boldsymbol{u}}_{h}, p-p_{h}, p-\hat{p}_{h} ; \delta_{u}, \delta_{\hat{u}}, \delta_{p}, \delta_{\hat{p}}\right) \\
= & \left(v \nabla_{h}\left(\boldsymbol{u}-\boldsymbol{u}_{h}\right), \nabla_{h} \delta_{u}\right)_{\mathcal{T}_{h}}+\left\langle\tau\left(\hat{\boldsymbol{u}}_{h}-\boldsymbol{u}_{h}\right), \delta_{u}-\delta_{\hat{u}}\right\rangle_{\partial \mathcal{T}_{h}}-\left\langle v \nabla_{h}\left(\boldsymbol{u}-\boldsymbol{u}_{h}\right) \boldsymbol{n}, \delta_{u}-\delta_{\hat{u}}\right\rangle_{\partial \mathcal{T}_{h}} \\
& -\left\langle\hat{\boldsymbol{u}}_{h}-\boldsymbol{u}_{h}, v\left(\nabla_{h} \delta_{u}\right) \boldsymbol{n}\right\rangle_{\partial \mathcal{T}_{h}}-\left(p-p_{h}, \nabla_{h} \cdot \delta_{u}\right) \mathcal{T}_{h}+\left\langle\left(p-\hat{p}_{h}\right) \boldsymbol{n}, \delta_{u}-\delta_{\hat{u}}\right\rangle_{\partial \mathcal{T}_{h}} \\
& +\left(\nabla_{h} \cdot\left(\boldsymbol{u}-\boldsymbol{u}_{h}\right), \delta_{p}\right)_{\mathcal{T}_{h}}-\left\langle\hat{\boldsymbol{u}}_{h}-\boldsymbol{u}_{h}, \delta_{\hat{p}} \boldsymbol{n}\right\rangle_{\partial \mathcal{T}_{h}} .
\end{aligned}
$$


Since the approximate velocity field is divergence-free, we have

$$
\nabla_{h} \cdot\left(\boldsymbol{u}-\boldsymbol{u}_{h}\right)=0
$$

From (2.9)-(2.10), we know that

$$
\left\langle\hat{\boldsymbol{u}}_{h}-\boldsymbol{u}_{h}, \delta_{\hat{p}} \boldsymbol{\eta}\right\rangle_{\partial \mathcal{T}_{h}}=0
$$

for HDG and E-HDG methods under the assumption that $p$ is continuous. Then integration by parts and the above two equalities result in

$$
\begin{aligned}
& \mathcal{B}\left(\boldsymbol{u}-\boldsymbol{u}_{h}, \boldsymbol{u}-\hat{\boldsymbol{u}}_{h}, p-p_{h}, p-\hat{p}_{h} ; \delta_{u}, \delta_{\hat{u}}, \delta_{p}, \delta_{\hat{p}}\right) \\
= & \left(\boldsymbol{f}+v \Delta \boldsymbol{u}_{h}-\nabla_{h} p_{h}, \delta_{u}\right)_{\mathcal{T}_{h}}+\left\langle\tau\left(\hat{\boldsymbol{u}}_{h}-\boldsymbol{u}_{h}\right), \delta_{u}-\delta_{\hat{u}}\right\rangle_{\partial \mathcal{T}_{h}}+\left\langle v \nabla_{h}\left(\boldsymbol{u}-\boldsymbol{u}_{h}\right) \boldsymbol{n}, \delta_{\hat{u}}\right\rangle_{\partial \mathcal{T}_{h}} \\
& -\left\langle\hat{\boldsymbol{u}}_{h}-\boldsymbol{u}_{h}, v\left(\nabla_{h} \delta_{u}\right) \boldsymbol{n}\right\rangle_{\partial \mathcal{T}_{h}}+\left\langle\left(p_{h}-\hat{p}_{h}\right) \boldsymbol{n}, \delta_{u}\right\rangle_{\partial \mathcal{T}_{h}}-\left\langle\left(p-\hat{p}_{h}\right) \boldsymbol{n}, \delta_{\hat{u}}\right\rangle_{\partial \mathcal{T}_{h}} .
\end{aligned}
$$

Hence

$$
\begin{aligned}
& \left\|v^{-\frac{1}{2}} \nabla_{h}\left(\boldsymbol{u}-\boldsymbol{u}_{h}\right)\right\|_{0, \mathcal{T}_{h}}^{2}+\left\|\tau^{\frac{1}{2}}\left(\boldsymbol{u}_{h}-\hat{\boldsymbol{u}}_{h}\right)\right\|_{0, \partial \mathcal{T}_{h}}^{2} \\
= & \mathcal{B}\left(\boldsymbol{u}-\boldsymbol{u}_{h}, \boldsymbol{u}-\hat{\boldsymbol{u}}_{h}, p-p_{h}, p-\hat{p}_{h} ; \delta_{u}, \delta_{\hat{u}}, \delta_{p}, \delta_{\hat{p}}\right)+2\left\langle\hat{\boldsymbol{u}}_{h}-\boldsymbol{u}_{h}, v \nabla_{h}\left(\boldsymbol{u}-\boldsymbol{u}_{h}\right) \boldsymbol{n}\right\rangle_{\partial \mathcal{T}_{h}} \\
= & \left(\boldsymbol{f}+v \Delta \boldsymbol{u}_{h}-\nabla_{h} p_{h}, \delta_{u}\right)_{\mathcal{T}_{h}} \\
& +\left\langle\boldsymbol{u}_{h}-\hat{\boldsymbol{u}}_{h}, v\left(\nabla_{h} \delta_{u}\right) \boldsymbol{n}\right\rangle_{\partial \mathcal{T}_{h}}+\left\langle\left(p_{h}-\hat{p}_{h}\right) \boldsymbol{n}, \delta_{u}\right\rangle_{\partial \mathcal{T}_{h}}-\left\langle\tau\left(\boldsymbol{u}_{h}-\hat{\boldsymbol{u}}_{h}\right), \delta_{u}\right\rangle_{\partial \mathcal{T}_{h}} \\
& +\left\langle v \nabla_{h}\left(\boldsymbol{u}-\boldsymbol{u}_{h}\right) \boldsymbol{n}, \delta_{\hat{u}}\right\rangle_{\partial \mathcal{T}_{h}}+\left\langle\tau\left(\boldsymbol{u}_{h}-\hat{\boldsymbol{u}}_{h}\right), \delta_{\hat{u}}\right\rangle_{\partial \mathcal{T}_{h}}-\left\langle\left(p-\hat{p}_{h}\right) \boldsymbol{n}, \delta_{\hat{u}}\right\rangle_{\partial \mathcal{T}_{h}} \\
& +2\left\langle\hat{\boldsymbol{u}}_{h}-\boldsymbol{u}_{h}, v \nabla_{h}\left(\boldsymbol{u}-\boldsymbol{u}_{h}\right) \boldsymbol{n}\right\rangle_{\partial \mathcal{T}_{h}} \\
= & \sum_{i=1}^{4} E_{i} .
\end{aligned}
$$

With Cauchy-Schwarz inequality and Lemma 3.6, we have

$$
\begin{aligned}
E_{1} & \leq \sum_{K \in \mathcal{T}_{h}} v^{-\frac{1}{2}}\left\|f+v \Delta \boldsymbol{u}_{h}-\nabla_{h} p_{h}\right\|_{0, K} v^{\frac{1}{2}}\left\|\delta_{u}\right\|_{0, K} \\
& \lesssim \sum_{K \in \mathcal{T}_{h}} \eta_{K} v^{\frac{1}{2}}\left\|\nabla \boldsymbol{u}-\nabla_{h} \boldsymbol{u}_{h}\right\|_{0, K} .
\end{aligned}
$$

Using (2.9)-(2.10), Lemma 3.3 and Lemma 3.7 to deduce that

$$
\begin{aligned}
E_{2}= & \left\langle\boldsymbol{u}_{h}-\hat{\boldsymbol{u}}_{h}, v\left(\nabla_{h} \delta_{u}\right) \boldsymbol{n}\right\rangle_{\partial \mathcal{T}_{h}}+\left\langle\left(p_{h}-\hat{p}_{h}\right) \boldsymbol{n}, \delta_{u}\right\rangle_{\partial \mathcal{T}_{h}}-\left\langle\tau\left(\boldsymbol{u}_{h}-\hat{\boldsymbol{u}}_{h}\right), \delta_{u}\right\rangle_{\partial \mathcal{T}_{h}} \\
= & \left\langle\boldsymbol{u}_{h}-\hat{\boldsymbol{u}}_{h},\left(v \nabla_{h} \delta_{u}-\left(p-p_{h}\right) \boldsymbol{I}\right) \boldsymbol{n}\right\rangle_{\partial \mathcal{T}_{h}}+\left\langle\boldsymbol{u}_{h}-\hat{\boldsymbol{u}}_{h},\left(\hat{p}_{h}-p_{h}\right) \boldsymbol{n}\right\rangle_{\partial \mathcal{T}_{h}} \\
& +\left\langle\left(p_{h}-\hat{p}_{h}\right) \boldsymbol{n}, \delta_{u}\right\rangle_{\partial \mathcal{T}_{h}}-\left\langle\tau\left(\boldsymbol{u}_{h}-\hat{\boldsymbol{u}}_{h}\right), \delta_{u}\right\rangle_{\partial \mathcal{T}_{h}}
\end{aligned}
$$




$$
\begin{aligned}
\leq & \sum_{K \in \mathcal{T}_{h}}\left\|h_{K}^{-\frac{1}{2}}\left(\boldsymbol{u}_{h}-\hat{\boldsymbol{u}}_{h}\right)\right\|_{0, \partial K}\left(\left\|v \nabla_{h}\left(\boldsymbol{u}-\boldsymbol{u}_{h}\right)\right\|_{0, K}+\left\|p-p_{h}\right\|_{0, K}\right) \\
& +\sum_{K \in \mathcal{T}_{h}}\left\|h_{K}^{-\frac{1}{2}}\left(\boldsymbol{u}_{h}-\hat{\boldsymbol{u}}_{h}\right)\right\|_{0, \partial K} h_{K}\left\|\nabla_{h} \cdot\left(v \nabla_{h}\left(\boldsymbol{u}-\boldsymbol{u}_{h}\right)\right)-\nabla_{h}\left(p-p_{h}\right)\right\|_{0, K} \\
& +\sum_{K \in \mathcal{T}_{h}}\left\|h_{K}^{-\frac{1}{2}}\left(\boldsymbol{u}_{h}-\hat{\boldsymbol{u}}_{h}\right)\right\|_{0, \partial K}\left(h_{K}^{-\frac{1}{2}} v\left\|\delta_{u}\right\|_{0, \partial K}+h_{K}^{\frac{1}{2}}\left\|p_{h}-\hat{p}_{h}\right\|_{0, \partial K}\right) \\
& +\left\|p_{h}-\hat{p}_{h}\right\|_{\partial \mathcal{T}_{h}}\left\|\delta_{u}\right\|_{\partial \mathcal{T}_{h}} \\
\leq & \sum_{K \in \mathcal{T}_{h}} \eta_{\partial K}\left(\left\|v^{\frac{1}{2}} \nabla_{h}\left(\boldsymbol{u}-\boldsymbol{u}_{h}\right)\right\|_{0, K}+v^{-\frac{1}{2}}\left\|p-p_{h}\right\|_{0, K}\right) \\
& \left.+\sum_{K \in \mathcal{T}_{h}} \eta_{\partial K}\left(v^{-\frac{1}{2}} h_{K} \| \boldsymbol{f}+v \Delta \boldsymbol{u}_{h}-\nabla_{h} p_{h}\right)\left\|_{0, K}+v^{-\frac{1}{2}} h_{K}^{\frac{1}{2}}\right\| p_{h}-\hat{p}_{h} \|_{0, \partial K}\right) \\
& +\sum_{K \in \mathcal{T}_{h}} h_{K}^{\frac{1}{2}}\left\|p_{h}-\hat{p}_{h}\right\|_{0, \partial K}\left\|\nabla_{h}\left(\boldsymbol{u}-\boldsymbol{u}_{h}\right)\right\|_{0, K} \\
\lesssim & \sum_{K \in \mathcal{T}_{h}}\left(\eta_{\partial K}+\eta_{K}\right)\left(\eta_{K}+\eta_{\partial K}+\left\|v^{\frac{1}{2}} \nabla_{h}\left(\boldsymbol{u}-\boldsymbol{u}_{h}\right)\right\|_{0, K}+v^{-\frac{1}{2}}\left\|p-p_{h}\right\|_{0, K}\right) .
\end{aligned}
$$

In HDG method, with the Cauchy-Schwarz inequality, (3.9) and (2.11) infer that

$$
\begin{aligned}
& E_{3}=\left\langle v \nabla_{h}\left(\boldsymbol{u}-\boldsymbol{u}_{h}\right) \boldsymbol{n}, \delta_{\hat{u}}\right\rangle_{\partial \mathcal{T}_{h}}+\left\langle\tau\left(\boldsymbol{u}_{h}-\hat{\boldsymbol{u}}_{h}\right), \delta_{\hat{u}}\right\rangle_{\partial \mathcal{T}_{h}}-\left\langle\left(p-\hat{p}_{h}\right) \boldsymbol{n}, \delta_{\hat{u}}\right\rangle_{\partial \mathcal{T}_{h}} \\
= & \left\langle v \nabla_{h}\left(\boldsymbol{u}-\boldsymbol{u}_{h}\right) \boldsymbol{n}-\left(p-p_{h}\right) \boldsymbol{n}+\tau\left(\boldsymbol{u}_{h}-\hat{\boldsymbol{u}}_{h}\right), \boldsymbol{u}-\hat{\boldsymbol{u}}_{h}\right\rangle_{\varepsilon_{h}^{\partial}}-\left\langle\left(p_{h}-\hat{p}_{h}\right) \boldsymbol{n}, \boldsymbol{u}-\hat{\boldsymbol{u}}_{h}\right\rangle_{\varepsilon_{h}^{\partial}} \\
\leq & \sum_{F \in \varepsilon_{h}^{\partial}}\left\|\boldsymbol{g}-\boldsymbol{I}_{h} \boldsymbol{g}\right\|_{1 / 2, F}\left(v\left\|\nabla_{h}\left(\boldsymbol{u}-\boldsymbol{u}_{h}\right)\right\|_{0, K_{F}}+\left\|p-p_{h}\right\|_{0, K_{F}}\right. \\
& \left.+h_{K_{F}}\left\|\nabla \cdot\left(v \nabla\left(\boldsymbol{u}-\boldsymbol{u}_{h}\right)-\left(p-p_{h}\right) \boldsymbol{I}\right)\right\|_{0, K_{F}}\right) \\
& +\sum_{F \in \varepsilon_{h}^{\partial}} h_{F}^{-1 / 2}\left\|\boldsymbol{g}-\boldsymbol{I}_{h} \boldsymbol{g}\right\|_{0, F}\left(v^{1 / 2}\left\|\tau^{1 / 2}\left(\boldsymbol{u}_{h}-\hat{\boldsymbol{u}}_{h}\right)\right\|_{0, F}+h_{F}^{1 / 2}\left\|p_{h}-\hat{p}_{h}\right\|_{0, F}\right) \\
\lesssim & O S c(\boldsymbol{g}, \partial \Omega)\left(v^{1 / 2}\left\|\nabla_{h}\left(\boldsymbol{u}-\boldsymbol{u}_{h}\right)\right\|_{0, \mathcal{T}_{h}}+v^{-1 / 2}\left\|p-p_{h}\right\|_{0, \mathcal{T}_{h}}+\left\{\sum_{K \in \mathcal{T}_{h}^{\partial}}\left(\eta_{K}^{2}+\eta_{\partial K}^{2}\right)\right\}^{1 / 2}\right) .
\end{aligned}
$$

In E-HDG method, taking $\boldsymbol{\mu}_{h}=\tilde{\boldsymbol{I}}_{h} \boldsymbol{u}-\tilde{\boldsymbol{I}}_{h} \tilde{\boldsymbol{I}}_{h}^{o s} \boldsymbol{u}_{h}+\tilde{\boldsymbol{I}}_{h}^{o s} \boldsymbol{u}_{h}-\hat{\boldsymbol{u}}_{h} \in \hat{\boldsymbol{V}}_{h}^{0}$,

$$
\begin{aligned}
& E_{3}=\left\langle v \nabla_{h}\left(\boldsymbol{u}-\boldsymbol{u}_{h}\right) \boldsymbol{n}, \delta_{\hat{u}}\right\rangle_{\partial \mathcal{T}_{h}}+\left\langle\tau\left(\boldsymbol{u}_{h}-\hat{\boldsymbol{u}}_{h}\right), \delta_{\hat{u}}\right\rangle_{\partial \mathcal{T}_{h}}-\left\langle\left(p-\hat{p}_{h}\right) \boldsymbol{n}, \delta_{\hat{u}}\right\rangle_{\partial \mathcal{T}_{h}} \\
= & \left\langle\left(-v \nabla_{h} \boldsymbol{u}_{h}+p_{h} \boldsymbol{I}\right) \boldsymbol{n}, \delta_{\hat{u}}\right\rangle_{\partial \mathcal{T}_{h}}+\left\langle\tau\left(\boldsymbol{u}_{h}-\hat{\boldsymbol{u}}_{h}\right), \delta_{\hat{u}}\right\rangle_{\partial \mathcal{T}_{h}}-\left\langle\left(p_{h}-\hat{p}_{h}\right) \boldsymbol{n}, \delta_{\hat{u}}\right\rangle_{\partial \mathcal{T}_{h}} \\
= & \left\langle\left(-v \nabla_{h} \boldsymbol{u}_{h}+p_{h} \boldsymbol{I}\right) \boldsymbol{n}+\tau\left(\boldsymbol{u}_{h}-\hat{\boldsymbol{u}}_{h}\right)-\left(p_{h}-\hat{p}_{h}\right) \boldsymbol{n} \boldsymbol{u}-\tilde{\boldsymbol{I}}_{h}^{o s} \boldsymbol{u}_{h}-\tilde{\boldsymbol{I}}_{h}\left(\boldsymbol{u}-\tilde{\boldsymbol{I}}_{h}^{o s} \boldsymbol{u}_{h}\right)\right\rangle_{\partial \mathcal{T}_{h}} .
\end{aligned}
$$

We note that

$$
\begin{aligned}
& \left\langle\left(-v \nabla_{h} \boldsymbol{u}_{h}+p_{h} \boldsymbol{I}\right) \boldsymbol{n}, \boldsymbol{u}-\tilde{\boldsymbol{I}}_{h}^{o s} \boldsymbol{u}_{h}-\tilde{\boldsymbol{I}}_{h}\left(\boldsymbol{u}-\tilde{\boldsymbol{I}}_{h}^{o s} \boldsymbol{u}_{h}\right)\right\rangle_{\partial \mathcal{T}_{h}} \\
\leq & \sum_{F \in \varepsilon_{h}^{i}} h_{F}^{\frac{1}{2}}\left\|\llbracket\left(v \nabla_{h} \boldsymbol{u}_{h}-p_{h} \boldsymbol{I}\right) \boldsymbol{n} \rrbracket\right\|_{0, F} \sum_{K \in \mathcal{T}_{h}}\left\|\nabla\left(\boldsymbol{u}-\tilde{\boldsymbol{I}}_{h}^{o s} \boldsymbol{u}_{h}\right)\right\|_{0, K} .
\end{aligned}
$$


So we have

$$
\begin{aligned}
E_{3} \lesssim & \sum_{F \in \varepsilon_{h}^{i}} h_{F}^{\frac{1}{2}}\left\|\llbracket\left(v \nabla_{h} \boldsymbol{u}_{h}-p_{h} \boldsymbol{I}\right) \boldsymbol{n} \rrbracket\right\|_{0, F} \sum_{K \in \mathcal{T}_{h}}\left\|\nabla\left(\boldsymbol{u}-\tilde{\boldsymbol{I}}_{h}^{o s} \boldsymbol{u}_{h}\right)\right\|_{0, K} \\
& +\sum_{K \in \mathcal{T}_{h}}\left(\left\|\tau^{\frac{1}{2}}\left(\boldsymbol{u}_{h}-\hat{\boldsymbol{u}}_{h}\right)\right\|_{0, \partial K}+v^{-\frac{1}{2}} h_{K}^{\frac{1}{2}}\left\|\left(p_{h}-\hat{p}_{h}\right)\right\|_{0, \partial K}\right) v^{\frac{1}{2}}\left\|\nabla\left(\boldsymbol{u}-\tilde{\boldsymbol{I}}_{h}^{o s} \boldsymbol{u}_{h}\right)\right\|_{0, K} \\
\lesssim & \sum_{K \in \mathcal{T}_{h}}\left(\eta_{J}+\eta_{\partial K}+v^{-\frac{1}{2}} h_{K}^{\frac{1}{2}}\left\|\left(p_{h}-\hat{p}_{h}\right)\right\|_{0, \partial K}\right) v^{\frac{1}{2}}\left(\left\|\nabla\left(\boldsymbol{u}-\boldsymbol{u}_{h}\right)\right\|_{0, K}+\left\|\nabla\left(\boldsymbol{u}_{h}-\tilde{\boldsymbol{I}}_{h}^{o s} \boldsymbol{u}_{h}\right)\right\|_{0, K}\right) .
\end{aligned}
$$

By using Lemma 3.5, we have

$$
v^{\frac{1}{2}} \sum_{K \in \mathcal{T}_{h}}\left\|\nabla\left(\boldsymbol{u}_{h}-\tilde{\boldsymbol{I}}_{h}^{o S} \boldsymbol{u}_{h}\right)\right\|_{0, K} \lesssim v^{\frac{1}{2}} \sum_{F \in \varepsilon_{h}^{i}} h_{F}^{-\frac{1}{2}}\left\|\llbracket \boldsymbol{u}_{h} \rrbracket\right\|_{0, F}^{2}+v^{\frac{1}{2}} \sum_{F \in \varepsilon_{h}^{\partial}} h_{F}^{-\frac{1}{2}}\left\|\boldsymbol{I}_{h} \boldsymbol{g}-\boldsymbol{u}_{h}\right\|_{0, F} \lesssim \sum_{K \in \mathcal{T}_{h}} \eta_{\partial K} \cdot
$$

Then Lemma 3.7 results in

$$
E_{3} \lesssim\left(\sum_{K \in \mathcal{T}_{h}}\left(\eta_{J}^{2}+\eta_{K}^{2}+\eta_{\partial K}^{2}\right)\right)^{1 / 2}\left(\left\|\nabla\left(\boldsymbol{u}-\boldsymbol{u}_{h}\right)\right\|_{0, \mathcal{T}_{h}}+\left(\sum_{K \in \mathcal{T}_{h}} \eta_{\partial K}^{2}\right)^{1 / 2}\right)
$$

Similarly, with (2.9)-(2.10) and Lemma 3.3, we have

$$
\begin{aligned}
E_{4}= & 2\left\langle\hat{\boldsymbol{u}}_{h}-\boldsymbol{u}_{h}, v \nabla_{h}\left(\boldsymbol{u}-\boldsymbol{u}_{h}\right) \boldsymbol{n}\right\rangle_{\partial \mathcal{T}_{h}} \\
= & 2\left\langle\hat{\boldsymbol{u}}_{h}-\boldsymbol{u}_{h}\left(v \nabla_{h}\left(\boldsymbol{u}-\boldsymbol{u}_{h}\right)-\left(p-p_{h}\right) \boldsymbol{I}\right) \boldsymbol{n}\right\rangle_{\partial \mathcal{T}_{h}}+2\left\langle\hat{\boldsymbol{u}}_{h}-\boldsymbol{u}_{h},\left(\hat{p}_{h}-p_{h}\right) \boldsymbol{n}\right\rangle_{\partial \mathcal{T}_{h}} \\
\lesssim & \sum_{K \in \mathcal{T}_{h}} v^{-\frac{1}{2}} \eta_{\partial K}\left(\left\|v \nabla_{h}\left(\boldsymbol{u}-\boldsymbol{u}_{h}\right)\right\|_{0, K}+\left\|p-p_{h}\right\|_{0, K}\right. \\
& \left.\quad+h_{K}\left\|\nabla_{h} \cdot\left(v \nabla_{h}\left(\boldsymbol{u}-\boldsymbol{u}_{h}\right)\right)-\nabla_{h}\left(p-p_{h}\right)\right\|_{0, K}+h_{K}^{\frac{1}{2}}\left\|p_{h}-\hat{p}_{h}\right\|_{0, \partial K}\right) \\
\lesssim & \sum_{K \in \mathcal{T}_{h}} \eta_{\partial K}\left(\left\|v^{\frac{1}{2}} \nabla_{h}\left(\boldsymbol{u}-\boldsymbol{u}_{h}\right)\right\|_{0, K}+v^{-\frac{1}{2}}\left\|p-p_{h}\right\|_{0, K}+\eta_{K}+\eta_{\partial K}\right) .
\end{aligned}
$$

Finally, with Hölder inequality, we can complete the proof.

Lemma 3.9. Let $(\boldsymbol{u}, p)$ and $\left(\boldsymbol{u}_{h}, \hat{\boldsymbol{u}}_{h}, p_{h}, \hat{p}_{h}\right)$ be the solutions of problem (1.1) and (2.5), respectively. Then we have

$$
v^{-\frac{1}{2}}\left\|p-p_{h}\right\|_{\mathcal{T}_{h}} \lesssim \eta+\left\|v^{\frac{1}{2}} \nabla_{h}\left(\boldsymbol{u}-\boldsymbol{u}_{h}\right)\right\|_{\mathcal{T}_{h}}
$$

Proof. Since $p-p_{h} \in L_{0}^{2}(\Omega)$, the inf-sup condition [5] implies

$$
\left\|p-p_{h}\right\|_{\mathcal{T}_{h}} \lesssim \sup _{\boldsymbol{v} \in \boldsymbol{H}_{0}^{1}(\Omega) \backslash\{\mathbf{0}\}} \frac{\left(p-p_{h}, \nabla \cdot \boldsymbol{v}\right)_{\mathcal{T}_{h}}}{\|\nabla \boldsymbol{v}\|_{\mathcal{T}_{h}}}
$$


Then with integration by parts and Eq. (1.1), we can obtain

$$
\begin{aligned}
& \left(p-p_{h}, \nabla \cdot \boldsymbol{v}\right)_{\mathcal{T}_{h}} \\
= & -\left(\nabla_{h}\left(p-p_{h}\right), \boldsymbol{v}\right)_{\mathcal{T}_{h}}+\left\langle\left(p-p_{h}\right) \boldsymbol{n}, \boldsymbol{v}\right\rangle_{\partial \mathcal{T}_{h}} \\
= & -\left(\boldsymbol{f}+v \Delta \boldsymbol{u}_{h}-\nabla_{h} p_{h}, \boldsymbol{v}\right)_{\mathcal{T}_{h}}+\left\langle\left(p-p_{h}\right) \boldsymbol{n}, \boldsymbol{v}\right\rangle_{\partial \mathcal{T}_{h}}-\left(\nabla_{h} \cdot\left(v \nabla_{h}\left(\boldsymbol{u}-\boldsymbol{u}_{h}\right)\right), \boldsymbol{v}\right)_{\mathcal{T}_{h}} \\
= & -\left(\boldsymbol{f}+v \Delta \boldsymbol{u}_{h}-\nabla_{h} p_{h}, \boldsymbol{v}\right)_{\mathcal{T}_{h}}+\left(v \nabla_{h}\left(\boldsymbol{u}-\boldsymbol{u}_{h}\right), \nabla_{h} \boldsymbol{v}\right)_{\mathcal{T}_{h}}-\left\langle v \nabla_{h}\left(\boldsymbol{u}-\boldsymbol{u}_{h}\right) \boldsymbol{n}, \boldsymbol{v}\right\rangle_{\partial \mathcal{T}_{h}} \\
& +\left\langle\left(p-p_{h}\right) \boldsymbol{n}, \boldsymbol{v}\right\rangle_{\partial \mathcal{T}_{h}} .
\end{aligned}
$$

By taking $\left(\boldsymbol{v}_{h}, \hat{\boldsymbol{\mu}}_{h}, q_{h}, \hat{q}_{h}\right)=\left(\boldsymbol{\Pi}_{\mathrm{BDM}} \boldsymbol{v}, \mathbf{0}, 0,0\right)$ in Eq. (2.5), we have

$$
\begin{aligned}
& \left(\boldsymbol{f}+v \Delta \boldsymbol{u}_{h}-\nabla_{h} p_{h}, \boldsymbol{\Pi}_{\mathrm{BDM}} \boldsymbol{v}\right)_{\mathcal{T}_{h}} \\
= & \left\langle\tau\left(\boldsymbol{u}_{h}-\hat{\boldsymbol{u}}_{h}\right), \boldsymbol{\Pi}_{\mathrm{BDM}} \boldsymbol{v}\right\rangle_{\partial \mathcal{T}_{h}}-\left\langle\left(p_{h}-\hat{p}_{h}\right) \boldsymbol{n}, \boldsymbol{\Pi}_{\mathrm{BDM}} \boldsymbol{v}\right\rangle_{\partial \mathcal{T}_{h}}-\left\langle\boldsymbol{u}_{h}-\hat{\boldsymbol{u}}_{h}, v \nabla_{h}\left(\boldsymbol{\Pi}_{\mathrm{BDM}} \boldsymbol{v}\right) \boldsymbol{n}\right\rangle_{\partial \mathcal{T}_{h}} .
\end{aligned}
$$

Then we can get

$$
\begin{aligned}
& \left(p-p_{h}, \nabla \cdot \boldsymbol{v}\right)_{\mathcal{T}_{h}} \\
= & -\left(\boldsymbol{f}+v \Delta \boldsymbol{u}_{h}-\nabla_{h} p_{h}, \boldsymbol{v}-\boldsymbol{\Pi}_{\mathrm{BDM}} \boldsymbol{v}\right)_{\mathcal{T}_{h}}+\left(v \nabla_{h}\left(\boldsymbol{u}-\boldsymbol{u}_{h}\right), \nabla_{h} \boldsymbol{v}\right)_{\mathcal{T}_{h}} \\
& -\left\langle v \nabla_{h}\left(\boldsymbol{u}-\boldsymbol{u}_{h}\right) \boldsymbol{n}, \boldsymbol{v}\right\rangle_{\partial \mathcal{T}_{h}}+\left\langle\left(p-p_{h}\right) \boldsymbol{n}, \boldsymbol{v}\right\rangle_{\partial \mathcal{T}_{h}}-\left\langle\tau\left(\boldsymbol{u}_{h}-\hat{\boldsymbol{u}}_{h}\right), \boldsymbol{\Pi}_{\mathrm{BDM}} \boldsymbol{v}\right\rangle_{\partial \mathcal{T}_{h}} \\
& +\left\langle\left(p_{h}-\hat{p}_{h}\right) \boldsymbol{n}, \boldsymbol{\Pi}_{\mathrm{BDM}} \boldsymbol{v}\right\rangle_{\partial \mathcal{T}_{h}}+\left\langle\boldsymbol{u}_{h}-\hat{\boldsymbol{u}}_{h}, v \nabla_{h}\left(\boldsymbol{\Pi}_{\mathrm{BDM}} \boldsymbol{v}\right) \boldsymbol{n}\right\rangle_{\partial \mathcal{T}_{h}} \\
= & -\left(\boldsymbol{f}+v \Delta \boldsymbol{u}_{h}-\nabla_{h} p_{h}, \boldsymbol{v}-\boldsymbol{\Pi}_{\mathrm{BDM}} \boldsymbol{v}\right)_{\mathcal{T}_{h}}+\left(v \nabla_{h}\left(\boldsymbol{u}-\boldsymbol{u}_{h}\right), \nabla_{h} \boldsymbol{v}\right)_{\mathcal{T}_{h}} \\
& -\left\langle v \nabla_{h}\left(\boldsymbol{u}-\boldsymbol{u}_{h}\right) \boldsymbol{n}, \boldsymbol{v}\right\rangle_{\partial \mathcal{T}_{h}}+\left\langle\left(p-\hat{p}_{h}\right) \boldsymbol{n}, \boldsymbol{v}\right\rangle_{\partial \mathcal{T}_{h}}-\left\langle\tau\left(\boldsymbol{u}_{h}-\hat{\boldsymbol{u}}_{h}\right), \boldsymbol{\Pi}_{\mathrm{BDM}} \boldsymbol{v}\right\rangle_{\partial \mathcal{T}_{h}} \\
& +\left\langle\left(p_{h}-\hat{p}_{h}\right) \boldsymbol{n}, \boldsymbol{\Pi}_{\mathrm{BDM}} \boldsymbol{v}-\boldsymbol{v}\right\rangle_{\partial \mathcal{T}_{h}}+\left\langle\boldsymbol{u}_{h}-\hat{\boldsymbol{u}}_{h}, v \nabla_{h}\left(\boldsymbol{\Pi}_{\mathrm{BDM}} \boldsymbol{v}\right) \boldsymbol{n}\right\rangle_{\partial \mathcal{T}_{h}} .
\end{aligned}
$$

For HDG method, from (2.11) and $\boldsymbol{v} \in \boldsymbol{H}_{0}^{1}(\Omega)$, we have

$$
-\left\langle v \nabla_{h}\left(\boldsymbol{u}-\boldsymbol{u}_{h}\right) \boldsymbol{n}, \boldsymbol{v}\right\rangle_{\partial \mathcal{T}_{h}}+\left\langle\left(p-\hat{p}_{h}\right) \boldsymbol{n}, \boldsymbol{v}\right\rangle_{\partial \mathcal{T}_{h}}=\left\langle\tau\left(\boldsymbol{u}_{h}-\hat{\boldsymbol{u}}_{h}\right), \boldsymbol{v}\right\rangle_{\partial \mathcal{T}_{h}} .
$$

Hence Lemma 3.6 results in

$$
\begin{aligned}
& \left(p-p_{h}, \nabla \cdot \boldsymbol{v}\right)_{\mathcal{T}_{h}} \\
= & -\left(\boldsymbol{f}+v \Delta \boldsymbol{u}_{h}-\nabla_{h} p_{h}, \boldsymbol{v}-\boldsymbol{\Pi}_{\mathrm{BDM}} \boldsymbol{v}\right)_{\mathcal{T}_{h}}+\left(v \nabla_{h}\left(\boldsymbol{u}-\boldsymbol{u}_{h}\right), \nabla_{h} \boldsymbol{v}\right)_{\mathcal{T}_{h}} \\
& +\left\langle\tau\left(\boldsymbol{u}_{h}-\hat{\boldsymbol{u}}_{h}\right)-\left(p_{h}-\hat{p}_{h}\right) \boldsymbol{n}, \boldsymbol{v}-\boldsymbol{\Pi}_{\mathrm{BDM}} \boldsymbol{v}\right\rangle_{\partial \mathcal{T}_{h}}+\left\langle\boldsymbol{u}_{h}-\hat{\boldsymbol{u}}_{h}, v \nabla_{h}\left(\boldsymbol{\Pi}_{\mathrm{BDM}} \boldsymbol{v}\right) \boldsymbol{n}\right\rangle_{\partial \mathcal{T}_{h}} \\
\lesssim & \sum_{K \in \mathcal{T}_{h}}\left(\eta_{K}+\left\|v^{\frac{1}{2}} \nabla_{h}\left(\boldsymbol{u}-\boldsymbol{u}_{h}\right)\right\|_{0, K}+\eta_{\partial K}\right) v^{\frac{1}{2}}\|\nabla \boldsymbol{v}\|_{0, K} .
\end{aligned}
$$

For E-HDG method, we can just get

$$
-\left\langle v \nabla_{h}\left(\boldsymbol{u}-\boldsymbol{u}_{h}\right) \boldsymbol{n}, \tilde{\boldsymbol{I}}_{h} \boldsymbol{v}\right\rangle_{\partial \mathcal{T}_{h}}+\left\langle\left(p-\hat{p}_{h}\right) \boldsymbol{n}, \tilde{\boldsymbol{I}}_{h} \boldsymbol{v}\right\rangle_{\partial \mathcal{T}_{h}}=\left\langle\tau\left(\boldsymbol{u}_{h}-\hat{\boldsymbol{u}}_{h}\right), \tilde{\boldsymbol{I}}_{h} \boldsymbol{v}\right\rangle_{\partial \mathcal{T}_{h}} .
$$


Then

$$
\begin{aligned}
& \left(p-p_{h}, \nabla \cdot \boldsymbol{v}\right)_{\mathcal{T}_{h}} \\
= & -\left(f-\left(-\nabla_{h} \cdot\left(v \nabla_{h} \boldsymbol{u}_{h}\right)+\nabla_{h} p_{h}\right), \boldsymbol{v}-\boldsymbol{\Pi}_{\mathrm{BDM}} \boldsymbol{v}\right)_{\mathcal{T}_{h}}+\left(v \nabla_{h}\left(\boldsymbol{u}-\boldsymbol{u}_{h}\right), \nabla_{h} \boldsymbol{v}\right)_{\mathcal{T}_{h}} \\
& -\left\langle v \nabla_{h}\left(\boldsymbol{u}-\boldsymbol{u}_{h}\right) \boldsymbol{n}, \boldsymbol{v}-\tilde{\boldsymbol{I}}_{h} \boldsymbol{v}\right\rangle_{\partial \mathcal{T}_{h}}+\left\langle\left(p-\hat{p}_{h}\right) \boldsymbol{n}, \boldsymbol{v}-\tilde{\boldsymbol{I}}_{h} \boldsymbol{v}\right\rangle_{\partial \mathcal{T}_{h}}+\left\langle\tau\left(\boldsymbol{u}_{h}-\hat{\boldsymbol{u}}_{h}\right), \tilde{\boldsymbol{I}}_{h} \boldsymbol{v}-\boldsymbol{\Pi}_{\mathrm{BDM}} \boldsymbol{v}\right\rangle_{\partial \mathcal{T}_{h}} \\
& +\left\langle\left(p_{h}-\hat{p}_{h}\right) \boldsymbol{n}, \boldsymbol{\Pi} \boldsymbol{\Pi}_{\mathrm{BDM}} \boldsymbol{v}-\boldsymbol{v}\right\rangle_{\partial \mathcal{T}_{h}}+\left\langle\boldsymbol{u}_{h}-\hat{\boldsymbol{u}}_{h}, v \nabla_{h}\left(\boldsymbol{\Pi}_{\mathrm{BDM}} \boldsymbol{v}\right) \boldsymbol{n}\right\rangle_{\partial \mathcal{T}_{h}} \\
= & -\left(\boldsymbol{f}-\left(-\nabla_{h} \cdot\left(v \nabla_{h} \boldsymbol{u}_{h}\right)+\nabla_{h} p_{h}\right), \boldsymbol{v}-\boldsymbol{\Pi}_{\mathrm{BDM}} \boldsymbol{v}\right)_{\mathcal{T}_{h}}+\left(v \nabla_{h}\left(\boldsymbol{u}-\boldsymbol{u}_{h}\right), \nabla_{h} \boldsymbol{v}\right)_{\mathcal{T}_{h}} \\
& -\left\langle v \nabla_{h}\left(\boldsymbol{u}-\boldsymbol{u}_{h}\right) \boldsymbol{n}, \boldsymbol{v}-\tilde{\boldsymbol{I}}_{h} \boldsymbol{v}\right\rangle_{\partial \mathcal{T}_{h}}+\left\langle\left(p-p_{h}\right) \boldsymbol{n}, \boldsymbol{v}-\tilde{\boldsymbol{I}}_{h} \boldsymbol{v}\right\rangle_{\partial \mathcal{T}_{h}}+\left\langle\tau\left(\boldsymbol{u}_{h}-\hat{\boldsymbol{u}}_{h}\right), \tilde{\boldsymbol{I}}_{h} \boldsymbol{v}-\boldsymbol{\Pi}_{\mathrm{BDM}} \boldsymbol{v}\right\rangle_{\partial \mathcal{T}_{h}} \\
& +\left\langle\left(p_{h}-\hat{p}_{h}\right) \boldsymbol{n}, \boldsymbol{\Pi}_{\mathrm{BDM}} \boldsymbol{v}-\tilde{\boldsymbol{I}}_{h} \boldsymbol{v}\right\rangle_{\partial \mathcal{T}_{h}}+\left\langle\boldsymbol{u}_{h}-\hat{\boldsymbol{u}}_{h}, v \nabla_{h}\left(\boldsymbol{\Pi}_{\mathrm{BDM}} \boldsymbol{v}\right) \boldsymbol{n}\right\rangle_{\partial \mathcal{T}_{h}} .
\end{aligned}
$$

With the fact that $v \nabla \boldsymbol{u}-p \boldsymbol{I} \in \boldsymbol{H}(\operatorname{div}, \Omega), \boldsymbol{v} \in \boldsymbol{H}_{0}^{1}(\Omega)$, and the definition of $\tilde{\boldsymbol{I}}_{h}$, one can obtain

$$
\begin{aligned}
& -\left\langle v \nabla_{h}\left(\boldsymbol{u}-\boldsymbol{u}_{h}\right) \boldsymbol{n}, \boldsymbol{v}-\tilde{\boldsymbol{I}}_{h} \boldsymbol{v}\right\rangle_{\partial \mathcal{T}_{h}}+\left\langle\left(p-p_{h}\right) \boldsymbol{n}, \boldsymbol{v}-\tilde{\boldsymbol{I}}_{h} \boldsymbol{v}\right\rangle_{\partial \mathcal{T}_{h}} \\
= & \sum_{F \in \varepsilon_{h}^{i}}\left\langle\llbracket v \nabla_{h}\left(\boldsymbol{u}-\boldsymbol{u}_{h}\right) \boldsymbol{n}-\left(p-p_{h}\right) \boldsymbol{n} \rrbracket, \boldsymbol{v}-\tilde{\boldsymbol{I}}_{h} \boldsymbol{v}\right\rangle_{0, F} \\
= & \sum_{F \in \varepsilon_{h}^{i}}\left\langle\llbracket\left(-v \nabla_{h} \boldsymbol{u}_{h}+p_{h} \boldsymbol{I}\right) \boldsymbol{n} \rrbracket, \boldsymbol{v}-\tilde{\boldsymbol{I}}_{h} \boldsymbol{v}\right\rangle_{0, F} \\
\lesssim & \sum_{K \in \mathcal{T}_{h}} h_{K}^{\frac{1}{2}}\left\|\llbracket\left(v \nabla_{h} \boldsymbol{u}_{h}-p_{h} \boldsymbol{I}\right) \boldsymbol{n} \rrbracket\right\|_{0, \partial K \backslash \varepsilon_{h}^{\partial}}\|\nabla \boldsymbol{v}\|_{0, K} .
\end{aligned}
$$

So we have

$$
\left(p-p_{h}, \nabla \cdot \boldsymbol{v}\right)_{\mathcal{T}_{h}} \lesssim \sum_{K \in \mathcal{T}_{h}}\left(\eta_{K}+\left\|v^{\frac{1}{2}} \nabla_{h}\left(\boldsymbol{u}-\boldsymbol{u}_{h}\right)\right\|_{0, K}+\eta_{\partial K}+\eta_{J}\right) v^{\frac{1}{2}}\|\nabla \boldsymbol{v}\|_{0, K}
$$

Finally, the inf-sup condition (3.18) concludes the proof.

According to the results in Lemma 3.8 and Lemma 3.9, we can obtain the following upper bound by choosing a suitable $\delta>0$.

Theorem 3.1. Let $(\boldsymbol{u}, p)$ and $\left(\boldsymbol{u}_{h}, \hat{\boldsymbol{u}}_{h}, p_{h}, \hat{p}_{h}\right)$ be the solutions of problem (1.1) and (2.5), respectively. We assume that the pressure $p$ is continuous, and $g=0$ for the E-HDG method. Then we have

$$
\begin{aligned}
& \left\|v^{\frac{1}{2}} \nabla_{h}\left(\boldsymbol{u}-\boldsymbol{u}_{h}\right)\right\|_{\mathcal{T}_{h}}+v^{-\frac{1}{2}}\left\|p-p_{h}\right\|_{\mathcal{T}_{h}} \lesssim \eta+o s c(\boldsymbol{g}, \partial \Omega), \\
& E_{u, p} \lesssim \eta+o s c(\boldsymbol{g}, \partial \Omega),
\end{aligned}
$$

where

$$
E_{u, p}=\left\|v^{\frac{1}{2}} \nabla_{h}\left(\boldsymbol{u}-\boldsymbol{u}_{h}\right)\right\|_{\mathcal{T}_{h}}+v^{-\frac{1}{2}}\left\|p-p_{h}\right\|_{\mathcal{T}_{h}}+\left\|\tau^{\frac{1}{2}}\left(\boldsymbol{u}_{h}-\hat{\boldsymbol{u}}_{h}\right)\right\|_{0, \partial \mathcal{T}_{h}}^{2}
$$




\subsection{Efficiency}

In this subsection, we prove that the a posteriori error estimator $\eta$ also provides a lower bound for the errors in the pressure and the velocity gradient. In order to achieve this, we introduce the element bubble functions by $B_{K}:=\prod_{i=1}^{d+1} \lambda_{i}$ for $K \in \mathcal{T}_{h}$, where $\lambda_{i}$ denotes the Lagrange basis function at $i$-th vertex in $K$. Moreover it holds some properties as follows.

Lemma 3.10 ([46, Lemma 3.3]). For each $K \in \mathcal{T}_{h}$, let $B_{K}$ be the bubble function on element $K$. Then for any $\boldsymbol{v} \in \boldsymbol{P}_{k}(K)$, we have

$$
\begin{aligned}
& \|\boldsymbol{v}\|_{0, K}^{2} \lesssim\left(\boldsymbol{v}, B_{K} \boldsymbol{v}\right)_{K^{\prime}} \\
& \left\|B_{K} \boldsymbol{v}\right\|_{0, K} \leq\|\boldsymbol{v}\|_{0, K} \\
& \left\|\nabla\left(B_{K} \boldsymbol{v}\right)\right\|_{0, K} \lesssim h_{K}^{-1}\|\boldsymbol{v}\|_{0, K} .
\end{aligned}
$$

In order to estimate the local error estimator $\eta_{J}$ for E-HDG method, we provide the following lemmas.

Lemma 3.11 ([1, Page 220, Theorem 7.23] and [5, Page 373, Proposition 14.1.5]). For given two pairs of Banach spaces $A_{i}$ and $B_{i}(i=0,1)$, and the maps $\mathcal{K}: A_{i} \rightarrow B_{i}$ is a bounded linear operator. Then it holds

$$
\mathcal{K}: A_{\theta, t} \rightarrow B_{\theta, t}
$$

Moreover,

$$
\|\mathcal{K}\|_{A_{\theta, t} \rightarrow B_{\theta, t}} \leq\|\mathcal{K}\|_{A_{0} \rightarrow B_{0}}^{1-\theta}\|\mathcal{K}\|_{A_{1} \rightarrow B_{1}}^{\theta}
$$

where $A_{\theta, t}:=\left[A_{0}, A_{1}\right]_{\theta, t}, B_{\theta, t}:=\left[B_{0}, B_{1}\right]_{\theta, t}$ for $1 \leq t \leq \infty$. The definitions of $A_{\theta, t}, B_{\theta, t}$ are referred to [5, Page 372].

Lemma 3.12 ([5, Page 374, Theorem 14.2.3]). Let $0<\theta<1$ and $1 \leq t \leq \infty$, if $D$ has a Lipschitz boundary, then

$$
W_{t}^{(1-\theta) k+\theta(k+1)}(D)=\left[W_{t}^{k}(D), W_{t}^{k+1}(D)\right]_{\theta, t}
$$

and the norms are equivalent.

Then we have the following inverse inequality on edge/face $F$ of element for real number.

Lemma 3.13. For real number $0<s<1$ and $s=(1-\theta) \times 0+\theta \times 1$, we have

$$
\left\|\boldsymbol{\mu}_{h}\right\|_{s, F} \lesssim h^{-s}\left\|\boldsymbol{\mu}_{h}\right\|_{0, F}, \quad \forall \boldsymbol{\mu}_{h} \in \boldsymbol{P}_{k}(F) .
$$

Proof. By taking $A_{0}=A_{1}=\boldsymbol{L}^{2}(F) \cap \boldsymbol{P}_{k}(F)$ and $B_{0}=\boldsymbol{L}^{2}(F) \cap \boldsymbol{P}_{k}(F), B_{1}=\boldsymbol{H}^{1}(F) \cap \boldsymbol{P}_{k}(F)$, set $\mathcal{K}: A_{i} \rightarrow B_{i}(i=0,1)$. Then with the standard inverse inequality, we have

$$
\left\|\mathcal{K} \boldsymbol{\mu}_{h}\right\|_{1, F}=\left\|\boldsymbol{\mu}_{h}\right\|_{1, F} \leq C_{1} h_{F}^{-1}\left\|\boldsymbol{\mu}_{h}\right\|_{0, F}
$$


which implies

$$
\|\mathcal{K}\|_{\mathbf{L}^{2}(F) \cap \boldsymbol{P}_{k}(F) \rightarrow \boldsymbol{H}^{1}(F) \cap \boldsymbol{P}_{k}(F)} \leq C_{1} h_{F}^{-1},
$$

and obviously, it holds

$$
\|\mathcal{K}\|_{\boldsymbol{L}^{2}(F) \cap \boldsymbol{P}_{k}(F) \rightarrow \boldsymbol{L}^{2}(F) \cap \boldsymbol{P}_{k}(F)} \leq C_{0},
$$

where $C_{0}, C_{1}$ are some bounded positive constants.

Then with Lemma 3.12 and Lemma 3.11, we have for any real number $0<\theta<1$, and $s=(1-\theta) \times 0+\theta \times 1$,

$$
\mathcal{K}: \boldsymbol{L}^{2}(F) \cap \boldsymbol{P}_{k}(F)=A_{\theta, 2} \rightarrow B_{\theta, 2}=\boldsymbol{H}^{s}(F) \cap \boldsymbol{P}_{k}(F),
$$

then with Lemma 3.11, it also holds

$$
\begin{aligned}
\|\mathcal{K}\|_{\boldsymbol{L}^{2} \cap \boldsymbol{P}_{k}(F) \rightarrow \boldsymbol{H}^{s} \cap \boldsymbol{P}_{k}(F)} & \leq\|\mathcal{K}\|_{L^{2} \cap \boldsymbol{P}_{k}(F) \rightarrow \boldsymbol{L}^{2} \cap \boldsymbol{P}_{k}(F)}^{1-\theta}\|\mathcal{K}\|_{L^{2} \cap \boldsymbol{P}_{k}(F) \rightarrow \boldsymbol{H}^{1} \cap \boldsymbol{P}_{k}(F)}^{\theta} \\
& \leq C_{0}^{1-\theta} C_{1}^{\theta} h_{F}^{-\theta} \leq C h_{F}^{-s} .
\end{aligned}
$$

Therefore,

$$
\begin{aligned}
\left\|\boldsymbol{\mu}_{h}\right\|_{s, F} & =\left\|\mathcal{K} \boldsymbol{\mu}_{h}\right\|_{s, F} \\
& \leq\|\mathcal{K}\|_{\boldsymbol{L}^{2}(F) \cap \boldsymbol{P}_{k}(F) \rightarrow \boldsymbol{H}^{s}(F) \cap \boldsymbol{P}_{k}(F)}\left\|\boldsymbol{\mu}_{h}\right\|_{0, F} \\
& \lesssim h_{F}^{-s}\left\|\boldsymbol{\mu}_{h}\right\|_{0, F}
\end{aligned}
$$

which complete the proof.

Remark 3.1. By taking $A_{0}=\boldsymbol{L}^{2}(F) \cap \boldsymbol{P}_{k}(F), A_{1}=\boldsymbol{H}^{1}(F) \cap \boldsymbol{P}_{k}(F)$ and $B_{0}=B_{1}=\boldsymbol{H}^{1}(F) \cap \boldsymbol{P}_{k}(F)$, and following the same routine in Lemma 3.13, we can also get

$$
\left\|\boldsymbol{\mu}_{h}\right\|_{1, F} \lesssim h^{s-1}\left\|\boldsymbol{\mu}_{h}\right\|_{s, F}, \quad \forall \boldsymbol{\mu}_{h} \in \boldsymbol{P}_{k}(F) \quad \text { and } \quad 0<s<1
$$

Lemma 3.14. For all $\boldsymbol{\mu}_{h} \in \boldsymbol{P}_{k}(F)$, we have

$$
h_{F}^{s}\left\|\boldsymbol{\mu}_{h}\right\|_{0, F} \lesssim\left\|\boldsymbol{\mu}_{h}\right\|_{-s, F}, \quad 0<s<1 .
$$

Proof. With Lemma3.13, we have

$$
\left\|\boldsymbol{\mu}_{h}\right\|_{-s, F}=\sup _{\boldsymbol{\mu} \in \boldsymbol{H}^{s}(F)} \frac{\left\langle\boldsymbol{\mu}_{h}, \boldsymbol{\mu}\right\rangle_{F}}{\|\boldsymbol{\mu}\|_{s, F}} \geq \frac{\left\langle\boldsymbol{\mu}_{h}, \boldsymbol{\mu}_{h}\right\rangle_{F}}{\left\|\boldsymbol{\mu}_{h}\right\|_{s, F}} \geq C \frac{\left\langle\boldsymbol{\mu}_{h}, \boldsymbol{\mu}_{h}\right\rangle_{F}}{h_{F}^{-s}\left\|\boldsymbol{\mu}_{h}\right\|_{0, F}} \geq C h_{F}^{s}\left\|\boldsymbol{\mu}_{h}\right\|_{0, F}
$$

This completes the proof.

Then we have the following results. 
Lemma 3.15. It hold the following estimations

$$
\begin{aligned}
& \eta_{K} \lesssim\left\|v^{\frac{1}{2}} \nabla_{h}\left(\boldsymbol{u}-\boldsymbol{u}_{h}\right)\right\|_{0, K}+v^{-\frac{1}{2}}\left\|p-p_{h}\right\|_{0, K}+o s c(f ; K) \\
& \left(\sum_{K \in \mathcal{T}_{h}} \eta_{J}^{2}\right)^{\frac{1}{2}} \lesssim\left\|v^{\frac{1}{2}} \nabla_{h}\left(\boldsymbol{u}-\boldsymbol{u}_{h}\right)\right\|_{\mathcal{T}_{h}}+v^{-\frac{1}{2}}\left\|p-p_{h}\right\|_{\mathcal{T}_{h}}+\operatorname{osc}\left(\boldsymbol{f} ; \mathcal{T}_{h}\right), \\
& \left(\sum_{K \in \mathcal{T}_{h}} \eta_{\partial K}^{2}\right)^{\frac{1}{2}} \lesssim E_{u, p} .
\end{aligned}
$$

Proof. We set $\boldsymbol{v}=\boldsymbol{P}_{h}^{k} \boldsymbol{f}-\left(-\nabla_{h} \cdot\left(v \nabla_{h} \boldsymbol{u}_{h}\right)+\nabla_{h} p_{h}\right)$, with triangular inequality, then we have

$$
\eta_{K} \leq \operatorname{osc}(f ; K)+v^{-\frac{1}{2}} h_{K}\|\boldsymbol{v}\|_{0, K},
$$

then with the Lemma 3.10 and integration by parts, we can obtain

$$
\begin{aligned}
& \|\boldsymbol{v}\|_{0, K}^{2} \lesssim\left(\boldsymbol{v}, B_{K} \boldsymbol{v}\right)_{K} \\
= & \left(\boldsymbol{P}_{h}^{k} \boldsymbol{f}-\boldsymbol{f}, B_{K} \boldsymbol{v}\right)_{K}-\left(\nabla \cdot\left(v \nabla_{h}\left(\boldsymbol{u}-\boldsymbol{u}_{h}\right)\right)-\nabla\left(p-p_{h}\right), B_{K} \boldsymbol{v}\right)_{K} \\
= & \left(\boldsymbol{P}_{h}^{k} \boldsymbol{f}-\boldsymbol{f}, B_{K} \boldsymbol{v}\right)_{K}+\left(v \nabla_{h}\left(\boldsymbol{u}-\boldsymbol{u}_{h}\right)-\left(p-p_{h}\right) \boldsymbol{I}, \nabla B_{K} \boldsymbol{v}\right)_{K} \\
\lesssim & \left.o s c(\boldsymbol{f} ; K) v^{\frac{1}{2}} h_{K}^{-1} \|_{0, K}\right)\left\|B_{K} \boldsymbol{v}\right\|_{0, K}+\left(\left\|v \nabla_{h}\left(\boldsymbol{u}-\boldsymbol{u}_{h}\right)\right\|_{0, K}+\left\|p-p_{h}\right\|_{0, K}\right)\left\|\nabla B_{K} \boldsymbol{v}\right\|_{0, K} \\
\lesssim & \left(\operatorname{osc}(\boldsymbol{f} ; K) v^{\frac{1}{2}} h_{K}^{-1}+h_{K}^{-1}\left\|v \nabla_{h}\left(\boldsymbol{u}-\boldsymbol{u}_{h}\right)\right\|_{0, K}+h_{K}^{-1}\left\|p-p_{h}\right\|_{0, K}\right)\|\boldsymbol{v}\|_{0, K}
\end{aligned}
$$

which yields the result of (3.28).

With the fact that $v \nabla \boldsymbol{u}-p \boldsymbol{I} \in \boldsymbol{H}(\operatorname{div}, \Omega)$, Lemma 3.14 and (3.9), yields

$$
\begin{aligned}
\sum_{K \in \mathcal{T}_{h}} \eta_{J}^{2} & \lesssim \sum_{F \in \varepsilon_{h}^{i}} v^{-1}\left\|\llbracket\left(v \nabla_{h} \boldsymbol{u}_{h}-p_{h} \boldsymbol{I}\right) \boldsymbol{n} \rrbracket\right\|_{-\frac{1}{2}, F}^{2} \\
& =\sum_{F \in \varepsilon_{h}^{i}} v^{-1}\left\|\llbracket\left(v \nabla_{h}\left(\boldsymbol{u}-\boldsymbol{u}_{h}\right)-\left(p-p_{h}\right) \boldsymbol{I}\right) \boldsymbol{n} \rrbracket\right\|_{-\frac{1}{2}, F}^{2} \\
& \lesssim \sum_{K \in \mathcal{T}_{h}} v^{-1}\left(\left\|\left(v \nabla_{h}\left(\boldsymbol{u}-\boldsymbol{u}_{h}\right)-\left(p-p_{h}\right) \boldsymbol{I}\right)\right\|_{0, K}^{2}+h_{K}^{2}\left\|\nabla_{h} \cdot\left(v \nabla_{h}\left(\boldsymbol{u}-\boldsymbol{u}_{h}\right)-\left(p-p_{h}\right) \boldsymbol{I}\right)\right\|_{0, K}^{2}\right) \\
& \lesssim \sum_{K \in \mathcal{T}_{h}}\left(\left\|v^{\frac{1}{2}} \nabla_{h}\left(\boldsymbol{u}-\boldsymbol{u}_{h}\right)\right\|_{0, K}^{2}+\left\|v^{-\frac{1}{2}}\left(p-p_{h}\right)\right\|_{0, K}^{2}+\eta_{K}^{2}\right),
\end{aligned}
$$

together with (3.28), we have (3.29).

Finally, together with the definition of $E_{u, p}$, we can get the result of (3.30). Then we complete the proof.

In light of Lemma 3.15 and the definition of estimator $\eta$, we have the following efficiency result for error estimator.

Theorem 3.2. Let $(\boldsymbol{u}, p)$ and $\left(\boldsymbol{u}_{h}, \hat{\boldsymbol{u}}_{h}, p_{h}, \hat{p}_{h}\right)$ be the solutions of problem (1.1) and (2.5), respectively. Then we have the following result

$$
\eta \lesssim E_{u, p}+\operatorname{osc}\left(f ; \mathcal{T}_{h}\right)
$$




\section{Numerical experiments}

In this section, we will give several examples to verify the performance of the obtained a posteriori error estimators by the following adaptive algorithm.

\section{Adaptive Algorithm}

Step 1. Given $\theta \in(0,1]$, the initial mesh $\mathcal{T}_{h}^{0}$, and the iteration number $\mathbf{M}$.

$$
\text { Set } i=0 \text {. }
$$

Step 2. Solve the system (2.5) on the mesh $\mathcal{T}_{h}^{i}$.

Step 3. Compute the error estimator $\eta$ defined in Section 3.

Step 4. Select the marking set $\mathcal{M}_{h} \subset \mathcal{T}_{h}$ such that

$$
\sum_{K \in \mathcal{M}_{h}} \eta_{K}^{2} \geq \theta \eta^{2}
$$

where $\eta_{K}$ is the restriction of $\eta$ on the element $K$.

\section{Step 5. If $i \geq \mathbf{M}$}

$$
\text { else }{ }^{\text {Stop }}
$$

Refine the marking set $\mathcal{M}_{h}$ by bisection to generate a new mesh $\mathcal{T}_{h}^{i+1}$.

Set $i=i+1$ and go back to Step 2 . end

Here we recall that in the implementation of numerical scheme (2.5) the penalty parameter is taken as $\alpha_{v}=6 k^{2}$ for HDG and as $\alpha_{v}=4 k^{2}$ for E-HDG method. Note that the convergence history is plotted in log-log coordinates in this section. And $e_{h}:=$ $v^{\frac{1}{2}}\left\|\nabla\left(\boldsymbol{u}-\boldsymbol{u}_{h}\right)\right\|_{\mathcal{T}_{h}}+v^{-\frac{1}{2}}\left\|p-p_{h}\right\|_{\mathcal{T}_{h}}$.

Example 4.1. We set $\Omega=(0,1)^{2}$. The analytical solution to the problem (1.1) is given by

$$
\begin{aligned}
& u_{1}(x, y)=-x^{2}(x-1)^{2} y(y-1)(2 y-1), \\
& u_{2}(x, y)=x(x-1)(2 x-1) y^{2}(y-1)^{2}, \\
& p(x, y)=x^{6}-y^{6},
\end{aligned}
$$

and the external body force $f$ and the boundary condition $g$ can be derived explicitly.

We adopt $N \times N$ uniform triangular meshes (c.f. Fig. 1 ) in this example. Table 1 shows the convergence histories for the error estimator $\eta$, the error $\boldsymbol{e}_{h}$, the effectiveness index $\eta / \boldsymbol{e}_{h}$, and the divergence of the approximate velocity $\left\|\nabla_{h} \cdot \boldsymbol{u}_{h}\right\|_{0, \mathcal{T}_{h}}$ with $v=1,10^{-3}$ and $k=1,2$. Then we get the following results:

- For HDG/E-HDG methods with different parameter $v$, the error estimator $\eta$ and the error $\boldsymbol{e}_{h}$ can get the convergence of order $k$ on the uniform meshes.

- The effectiveness index $\frac{\eta}{e_{h}}$ is in the intervals $[5,9]$ for $k=1$ and $[12,15]$ for $k=2$. 
Table 1: The error estimator $\eta$, the error $e_{h}$, the effectiveness index $\eta / e_{h}$ and the divergence of the approximate velocity $\left\|\nabla_{h} \cdot \boldsymbol{u}_{h}\right\|_{0, \mathcal{T}_{h}}$ for different $v$.

(a) $v=1$

\begin{tabular}{|c|c|c|c|c|c|c|c|c|}
\hline method & $k$ & mesh & $\eta$ & order & $\overline{e_{h}}$ & order & $\eta / e_{h}$ & $\left\|\nabla_{h} \cdot \boldsymbol{u}_{h}\right\|_{0}$ \\
\hline \multirow{12}{*}{ HDG } & \multirow{6}{*}{$k=1$} & $4 \times 4$ & $9.0691 \mathrm{E}-01$ & - & $1.4715 \mathrm{E}-01$ & - & 6.16 & $3.4694 \mathrm{E}-17$ \\
\hline & & $8 \times 8$ & 4.5451E-01 & 1.00 & $7.9856 \mathrm{E}-02$ & 0.88 & 5.69 & 3.3168E-15 \\
\hline & & $16 \times 16$ & 2.2731E-01 & 1.00 & 4.0827E-02 & 0.97 & 5.57 & $6.9389 \mathrm{E}-17$ \\
\hline & & $32 \times 32$ & $1.1365 \mathrm{E}-01$ & 1.00 & 2.0474E-02 & 1.00 & 5.55 & 6.5364E-15 \\
\hline & & $64 \times 64$ & 5.6819E-02 & 1.00 & $1.0223 \mathrm{E}-02$ & 1.00 & 5.56 & 4.4728E-14 \\
\hline & & $128 \times 128$ & $2.8408 \mathrm{E}-02$ & 1.00 & $5.1074 \mathrm{E}-03$ & 1.00 & 5.56 & $6.7143 \mathrm{E}-12$ \\
\hline & \multirow{6}{*}{$k=2$} & $4 \times 4$ & $2.9283 \mathrm{E}-01$ & - & $2.2916 \mathrm{E}-02$ & - & 12.78 & $6.9670 \mathrm{E}-16$ \\
\hline & & $8 \times 8$ & 7.6052E-02 & 1.95 & $6.1319 \mathrm{E}-03$ & 1.90 & 12.40 & 2.3611E-15 \\
\hline & & $16 \times 16$ & 1.9181E-02 & 1.99 & $1.5583 \mathrm{E}-03$ & 1.98 & 12.31 & $1.9324 \mathrm{E}-15$ \\
\hline & & $32 \times 32$ & 4.8043E-03 & 2.00 & 3.9093E-04 & 2.00 & 12.29 & $8.2265 \mathrm{E}-14$ \\
\hline & & $64 \times 64$ & 1.2015E-03 & 2.00 & $9.7782 \mathrm{E}-05$ & 2.00 & 12.29 & $2.4841 \mathrm{E}-13$ \\
\hline & & $128 \times 128$ & $3.0038 \mathrm{E}-04$ & 2.00 & $2.4444 \mathrm{E}-05$ & 2.00 & 12.29 & $2.7464 \mathrm{E}-12$ \\
\hline \multirow{12}{*}{ E-HDG } & \multirow{6}{*}{$k=1$} & $4 \times 4$ & $1.1326 \mathrm{E}-00$ & - & $1.4696 \mathrm{E}-01$ & - & 7.71 & $1.3878 \mathrm{E}-17$ \\
\hline & & $8 \times 8$ & $6.3135 \mathrm{E}-01$ & 0.84 & 7.9458E-02 & 0.89 & 7.96 & $4.1633 \mathrm{E}-17$ \\
\hline & & $16 \times 16$ & 3.3910E-01 & 0.90 & 4.0506E-02 & 0.97 & 8.37 & 3.4694E-17 \\
\hline & & $32 \times 32$ & 1.7637E-01 & 0.94 & $2.0349 \mathrm{E}-02$ & 0.99 & 8.67 & $1.9082 \mathrm{E}-15$ \\
\hline & & $64 \times 64$ & $9.0050 \mathrm{E}-02$ & 0.97 & $1.0186 \mathrm{E}-02$ & 1.00 & 8.84 & 9.2093E-14 \\
\hline & & $128 \times 128$ & 4.5513E-02 & 0.98 & $5.0945 \mathrm{E}-03$ & 1.00 & 8.93 & $1.0494 \mathrm{E}-12$ \\
\hline & \multirow{6}{*}{$k=2$} & $4 \times 4$ & $3.3116 \mathrm{E}-01$ & - & $2.3030 \mathrm{E}-02$ & - & 14.38 & $5.3296 \mathrm{E}-16$ \\
\hline & & $8 \times 8$ & $8.6548 \mathrm{E}-02$ & 1.94 & $6.2142 \mathrm{E}-03$ & 1.89 & 13.93 & 1.4157E-15 \\
\hline & & $16 \times 16$ & 2.2039E-02 & 1.97 & $1.5901 \mathrm{E}-03$ & 1.97 & 13.86 & 2.6709E-15 \\
\hline & & $32 \times 32$ & $5.5689 \mathrm{E}-03$ & 1.98 & 4.0037E-04 & 1.99 & 13.91 & $8.5888 \mathrm{E}-13$ \\
\hline & & $64 \times 64$ & $1.4011 \mathrm{E}-03$ & 1.99 & $1.0030 \mathrm{E}-04$ & 2.00 & 13.97 & 5.7137E-13 \\
\hline & & $128 \times 128$ & 3.5152E-04 & 1.99 & $2.5088 \mathrm{E}-05$ & 2.00 & 14.01 & $1.3878 \mathrm{E}-12$ \\
\hline
\end{tabular}

(b) $v=10^{-3}$

\begin{tabular}{|c|c|c|c|c|c|c|c|c|}
\hline method & $k$ & mesh & $\eta$ & order & $e_{h}$ & order & $\eta / e_{h}$ & $\nabla_{h} \cdot \boldsymbol{u}_{h} \|_{0}$ \\
\hline \multirow{12}{*}{ HDG } & \multirow{6}{*}{$k=1$} & $4 \times 4$ & $2.8536 \mathrm{E}+01$ & - & $4.6104 \mathrm{E}-00$ & - & 6.19 & $1.1258 \mathrm{E}-13$ \\
\hline & & $8 \times 8$ & $1.4300 \mathrm{E}+01$ & 1.00 & $2.4926 \mathrm{E}-00$ & 0.89 & 5.74 & $2.3559 \mathrm{E}-13$ \\
\hline & & $16 \times 16$ & 7.1509E-00 & 1.00 & $1.2712 \mathrm{E}-00$ & 0.97 & 5.63 & $9.1036 \mathrm{E}-13$ \\
\hline & & $32 \times 32$ & $3.5755 \mathrm{E}-00$ & 1.00 & 6.3876E-01 & 0.99 & 5.60 & $2.2146 \mathrm{E}-13$ \\
\hline & & $64 \times 64$ & 1.7877E-00 & 1.00 & 3.1978E-01 & 1.00 & 5.59 & 7.3217E-10 \\
\hline & & $128 \times 128$ & 8.9387E-01 & 1.00 & $1.5994 \mathrm{E}-01$ & 1.00 & 5.59 & $2.0141 \mathrm{E}-13$ \\
\hline & \multirow{6}{*}{$k=2$} & $4 \times 4$ & $9.2037 \mathrm{E}-00$ & - & $6.9264 \mathrm{E}-01$ & - & 13.29 & $4.9460 \mathrm{E}-14$ \\
\hline & & $8 \times 8$ & 2.3898E-00 & 1.95 & $1.8511 \mathrm{E}-01$ & 1.90 & 12.91 & $9.7659 \mathrm{E}-13$ \\
\hline & & $16 \times 16$ & $6.0322 \mathrm{E}-01$ & 1.99 & $4.7048 \mathrm{E}-02$ & 1.98 & 12.82 & $1.2628 \mathrm{E}-12$ \\
\hline & & $32 \times 32$ & 1.5117E-01 & 2.00 & 1.1811E-02 & 1.99 & 12.80 & $7.5910 \mathrm{E}-12$ \\
\hline & & $64 \times 64$ & $3.7814 \mathrm{E}-02$ & 2.00 & 2.9557E-03 & 2.00 & 12.79 & $9.7546 \mathrm{E}-12$ \\
\hline & & $128 \times 128$ & $9.4550 \mathrm{E}-03$ & 2.00 & 7.3911E-04 & 2.00 & 12.79 & $1.1251 \mathrm{E}-10$ \\
\hline \multirow{12}{*}{ E-HDG } & \multirow{6}{*}{$k=1$} & $4 \times 4$ & $3.5356 \mathrm{E}+01$ & - & $4.6104 \mathrm{E}-00$ & - & 7.67 & $3.0212 \mathrm{E}-14$ \\
\hline & & $8 \times 8$ & $1.9783 \mathrm{E}+01$ & 0.84 & $2.4926 \mathrm{E}-00$ & 0.89 & 7.94 & $2.6809 \mathrm{E}-13$ \\
\hline & & $16 \times 16$ & $1.0621 \mathrm{E}+01$ & 0.90 & $1.2712 \mathrm{E}-00$ & 0.97 & 8.36 & $1.8839 \mathrm{E}-12$ \\
\hline & & $32 \times 32$ & $5.5283 \mathrm{E}-00$ & 0.94 & 6.3876E-01 & 0.99 & 8.65 & $2.2524 \mathrm{E}-11$ \\
\hline & & $64 \times 64$ & $2.8237 \mathrm{E}-00$ & 0.97 & $3.1978 \mathrm{E}-01$ & 1.00 & 8.83 & $1.5396 \mathrm{E}-12$ \\
\hline & & $128 \times 128$ & $1.4274 \mathrm{E}-00$ & 0.98 & $1.5994 \mathrm{E}-01$ & 1.00 & 8.93 & 1.3203E-10 \\
\hline & \multirow{6}{*}{$k=2$} & $4 \times 4$ & $1.0264 \mathrm{E}+01$ & - & $6.9264 \mathrm{E}-01$ & - & 14.82 & $4.0106 \mathrm{E}-14$ \\
\hline & & $8 \times 8$ & 2.6634E-00 & 1.95 & 1.8511E-01 & 1.90 & 14.39 & 7.1093E-13 \\
\hline & & $16 \times 16$ & $6.7652 \mathrm{E}-01$ & 1.98 & $4.7048 \mathrm{E}-02$ & 1.98 & 14.38 & 7.8953E-13 \\
\hline & & $32 \times 32$ & $1.7072 \mathrm{E}-01$ & 1.99 & $1.1811 \mathrm{E}-02$ & 1.99 & 14.46 & 4.1957E-12 \\
\hline & & $64 \times 64$ & 4.2912E-02 & 1.99 & 2.9557E-03 & 2.00 & 14.52 & 5.1149E-12 \\
\hline & & $128 \times 128$ & $1.0760 \mathrm{E}-02$ & 2.00 & 7.3911E-04 & 2.00 & 14.56 & $9.4008 \mathrm{E}-11$ \\
\hline
\end{tabular}




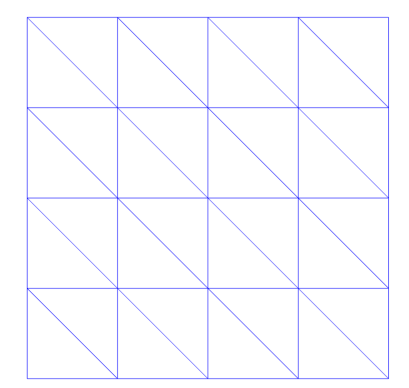

Figure 1: Regular triangular meshes: $4 \times 4$ mesh.

- The divergence of the approximate velocity is almost zero, which shows that HDG and E-HDG methods are pointwise divergence-free.

Example 4.2. We set $\Omega=(0,1)^{2}$. The analytical solution to the problem (1.1) is given by

$$
\begin{aligned}
& u_{1}(x, y)=5 x y^{4}-x^{5}, \\
& u_{2}(x, y)=5 x^{4} y-y^{5}, \\
& p(x, y)=\frac{1}{3}\left(x^{3}-y^{3}\right),
\end{aligned}
$$

then the force term $f$ and boundary condition $g$ can be derived explicitly.

The initial mesh is taken as Fig. 1. We plot the convergence histories of $\eta$ and $\boldsymbol{e}_{h}$ and the effectiveness index $\eta / e_{h}$ for different $k$ and $v$ in Figs. 2-3, which shows that the adaptive algorithm can get the convergence rate $\mathcal{O}\left(N^{-k / 2}\right)$ for both the error estimator $\eta$ and the error $\boldsymbol{e}_{h}$.

Example 4.3. We set $\Omega=(0,1)^{2}$. The analytical solution to the problem (1.1) is given by

$$
\begin{aligned}
& u_{1}(x, y)=y-\frac{1-e^{y / v}}{1-e^{1 / v}} \\
& u_{2}(x, y)=x-\frac{1-e^{x / v}}{1-e^{1 / v}} \\
& p(x, y)=x^{6}-y^{6}
\end{aligned}
$$

then the force term $f$ and boundary condition $g$ can be derived explicitly. Note that this example possesses the boundary layers on the boundaries $x=1$ and $y=1$.

The initial mesh is taken as Fig. 1. Then we have the following conclusions:

- The adaptive meshes after 25 iterations are presented in Fig. 4 for $\theta=0.5$. We can observe that the mesh nodes are concentrated around the boundary layers, which means that the obtained a posteriori error estimator can grad the boundary layers well. 

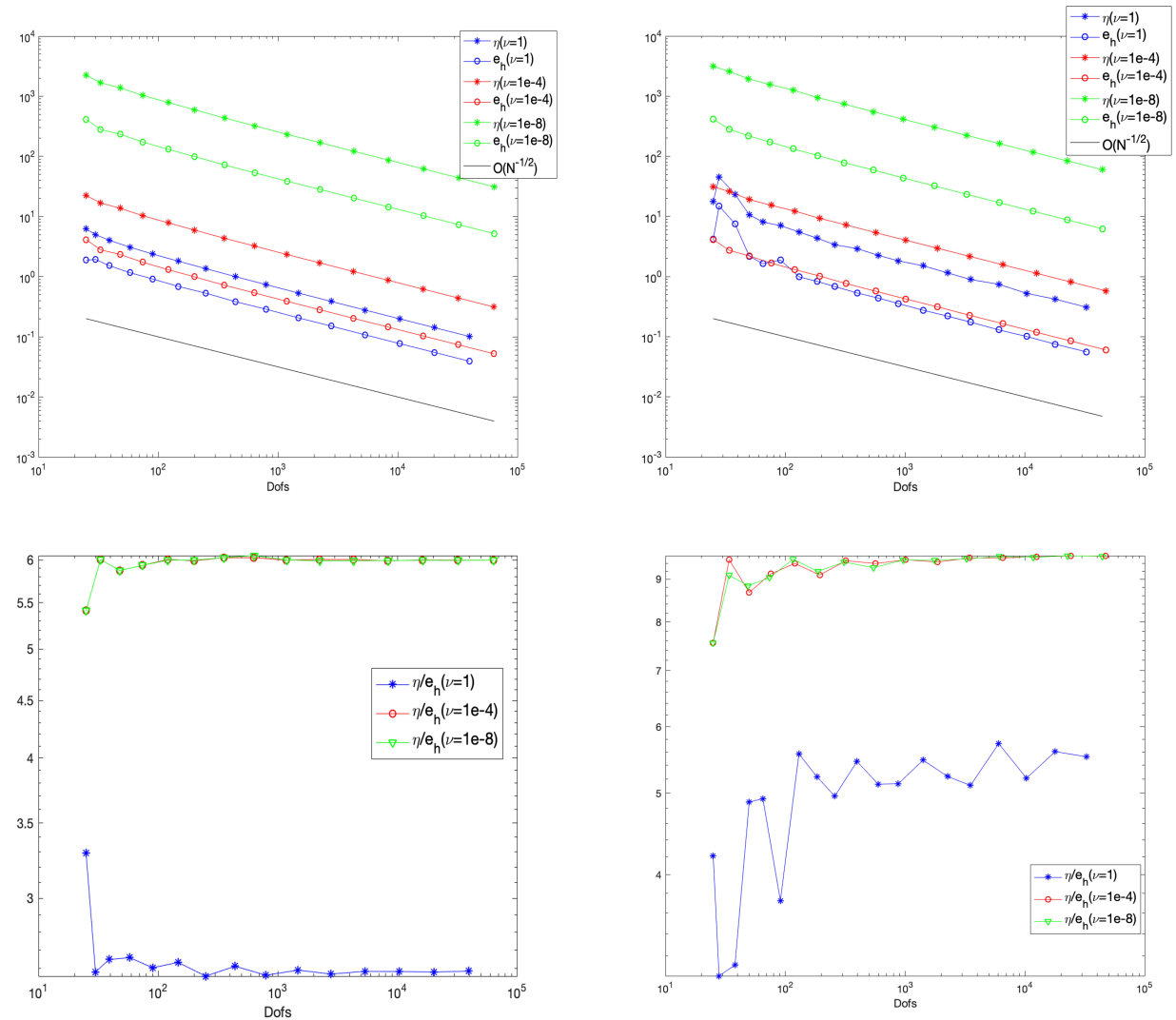

Figure 2: Here we set $v=1,1 e-4,1 e-8, k=1$ and $\theta=0.5$. Top: The convergence history of $\eta, e_{h}$ for HDG (left) and E-HDG (right). Bottom: The effectiveness index $\eta / e_{h}$ for HDG (left) and E-HDG (right).

- The convergence histories of the error estimator $\eta$ and the error $\boldsymbol{e}_{h}$ are provided in Fig. 5 for $k=1,2$ and $v=0.005$. Obviously, the error estimator and the error can achieve the convergence rate $\mathcal{O}\left(N^{-k / 2}\right)$. Moreover, seemingly, the numerical error has not significantly difference for HDG and E-HDG methods. Hence the E-HDG method outperforms the HDG method in the sense that the number of nodes for the E-HDG method is smaller than the HDG method.

Example 4.4. We set $\Omega=(-1,1)^{2} \backslash[0,1) \times(-1,0], f=0$ and $v=1$. The analytical solution to the problem (1.1) in polar coordinates $(r, \varphi)$ is

$$
\begin{aligned}
& u_{1}(r, \varphi)=r^{\lambda}\left[(1+\lambda) \sin (\varphi) \Psi(\varphi)+\cos (\varphi) \Psi^{\prime}(\varphi)\right], \\
& u_{2}(r, \varphi)=r^{\lambda}\left[\sin (\varphi) \Psi^{\prime}(\varphi)-(1+\lambda) \cos (\varphi) \Psi(\varphi)\right], \\
& p(r, \varphi)=-r^{\lambda-1}\left[(1+\lambda)^{2} \Psi^{\prime}(\varphi)+\Psi^{\prime \prime \prime}(\varphi)\right] /(1-\lambda),
\end{aligned}
$$



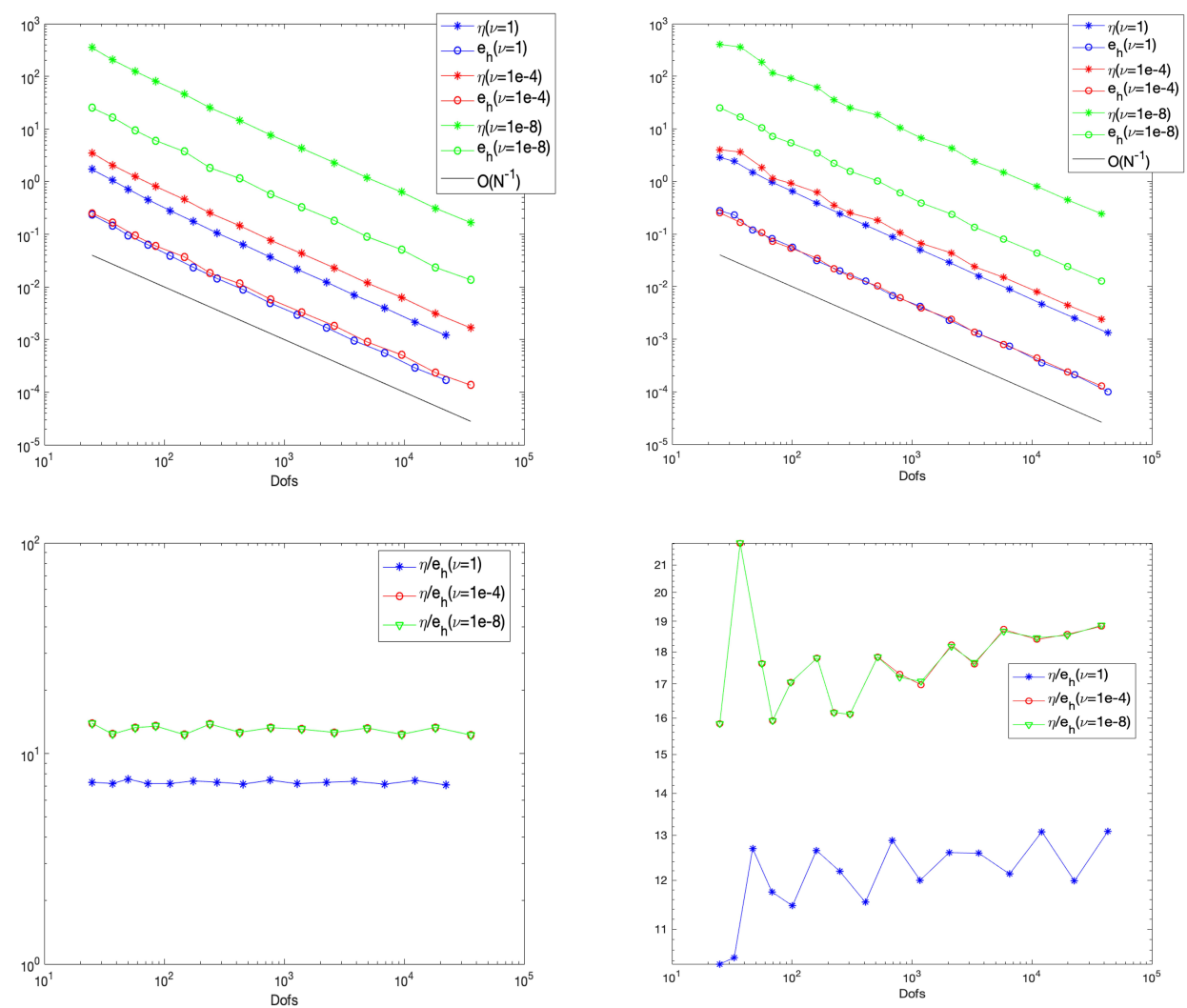

Figure 3: Here we set $v=1,1 e-4,1 e-8, k=2$ and $\theta=0.5$. Top: The convergence history of $\eta, e_{h}$ for HDG (left) and E-HDG (right). Bottom: The effectiveness index $\eta / e_{h}$ for HDG (left) and E-HDG (right).
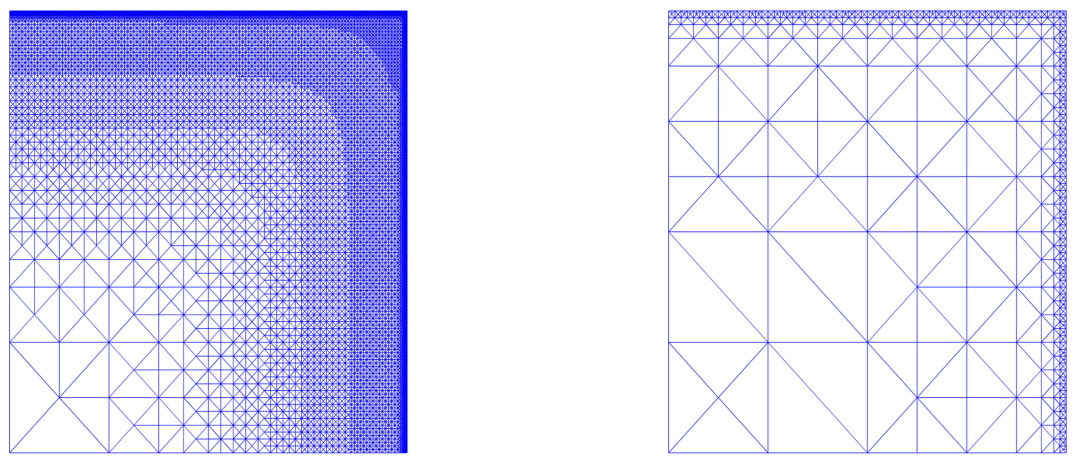

Figure 4: Adaptive meshes after 23 iterations: HDG $k=1$ (left) and $k=2$ (right). 

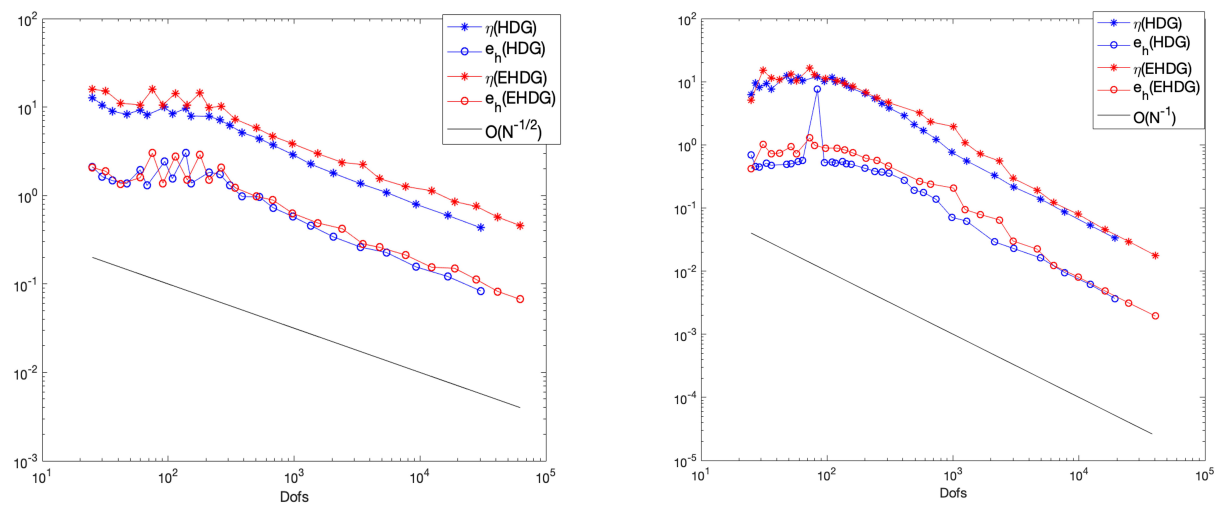

Figure 5: The convergence history of Example 4.3 with $v=0.005, \theta=0.5: k=1$ (left) and $k=2$ (right).
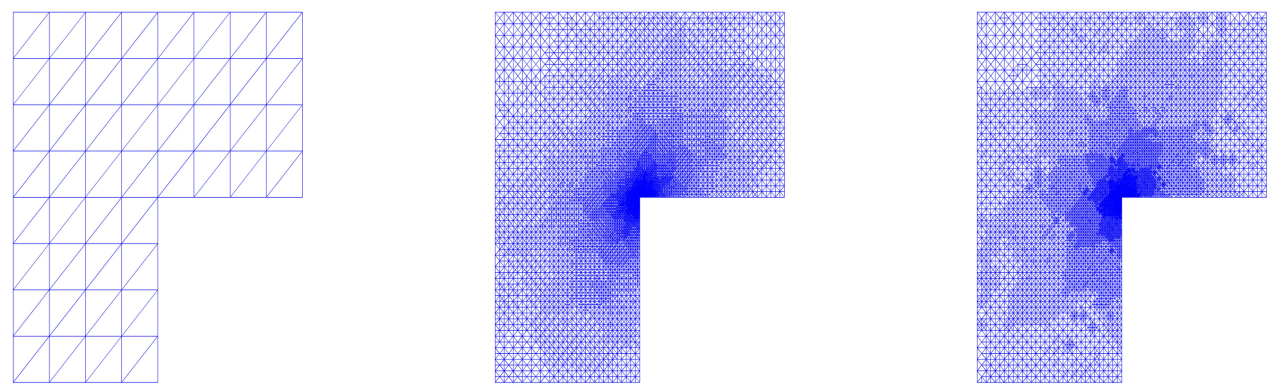

Figure 6: The initial mesh (left) and the adaptive meshes after 20 iteration for HDG (middle) and E-HDG (right) methods. Here we set $k=1$ and $\theta=0.5$.

where

$$
\begin{aligned}
\Psi(\varphi)= & \sin ((1+\lambda) \varphi) \cos (\lambda \omega) /(1+\lambda)-\cos ((1+\lambda) \varphi) \\
& -\sin ((1-\lambda) \varphi) \cos (\lambda \omega) /(1-\lambda)+\cos ((1-\lambda) \varphi),
\end{aligned}
$$

with the parameters $\lambda=0.54448373678246$ and $\omega=3 \pi / 2$. The boundary condition $g$ can be derived explicitly.

Note that the velocity gradient $\nabla \boldsymbol{u}$ and the pressure $p$ are singular at the original point. Indeed, $\boldsymbol{u} \notin\left(H^{2}(\Omega)\right)^{2}$ and $p \notin H^{1}(\Omega)$. For this example, we have the following results:

- Fig. 6 shows the initial mesh and the adaptive meshes after 20 iterations for HDG and E-HDG methods. Obviously, the mesh nodes are concentrated around the original point. Moreover, the effect of refinement has not significantly difference for HDG and E-HDG methods. 

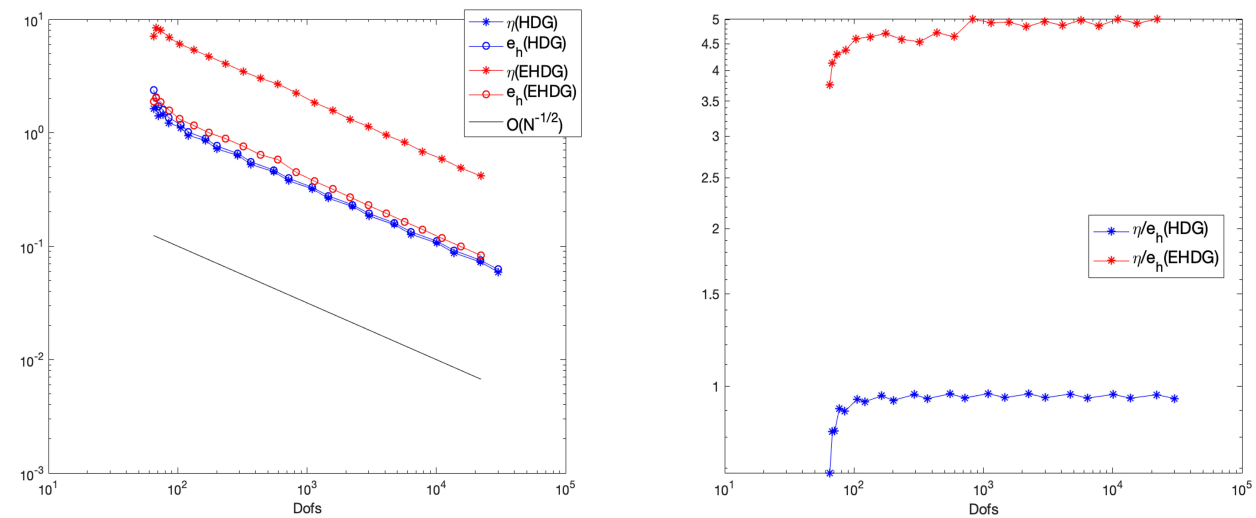

Figure 7: The convergence history (left) and effectiveness index $\eta / e_{h}$ (right) for $\theta=0.5$.
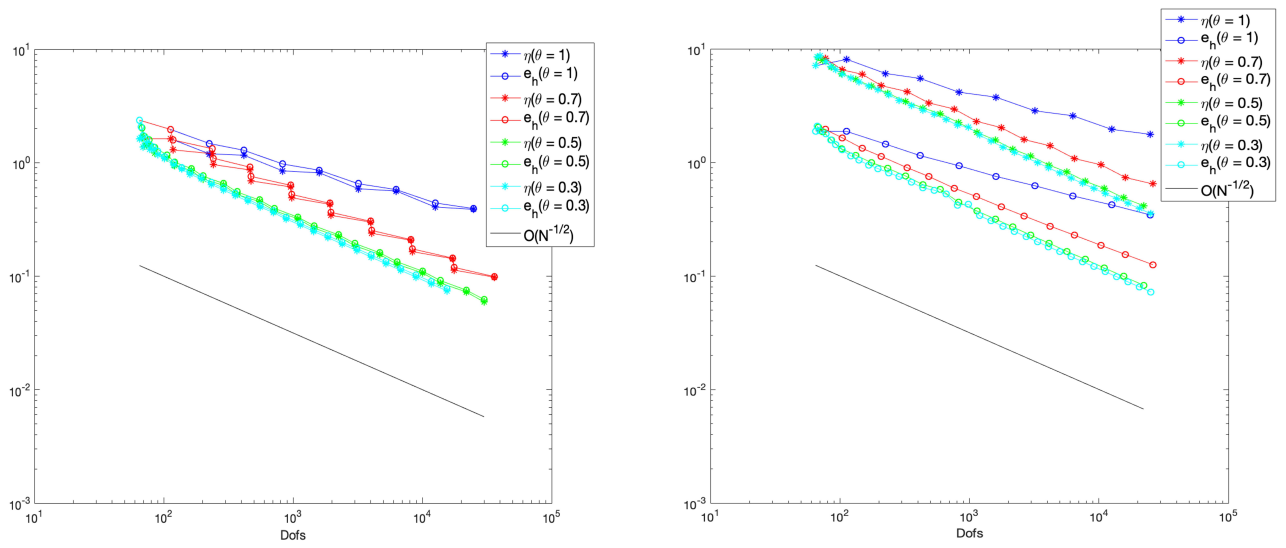

Figure 8: The convergence history with $\theta=1,0.7,0.5,0.3$ for HDG (left) and EHDG (right) methods.

- The convergence histories of the error estimator $\eta$ and the error $\boldsymbol{e}_{h}$ and the effectiveness index $\eta / e_{h}$ are given in Fig. 7 for $\theta=0.5$, which shows that the adaptive refinement achieves the convergence rate $\mathcal{O}\left(N^{-1 / 2}\right)$ for the error and the error estimator for HDG and E-HDG methods.

- In Fig. 8, we plot the convergence histories of the error estimator $\eta$ and the error $\boldsymbol{e}_{h}$ for different $\theta$, which shows that for both HDG and E-HDG methods, the error estimator $\eta$ and the error $\boldsymbol{e}_{h}$ can get the convergence rate $\mathcal{O}\left(N^{-1 / 2}\right)$ if $\theta$ is less than a suitable value. Moreover, we find that the adaptive refinement outperforms the uniform refinement. 


\section{Conclusions}

In this paper, we proposed a posteriori error estimator of embedded-hybridized Discontinuous Galerkin methods for the Stokes equations. The attractive properties, named divergence-free and $\boldsymbol{H}$ (div)-conforming, can be obtained for the approximate velocity field. Moreover, the pressure-robustness, which means that a priori error estimates for the velocity is independent of the pressure, is also satisfied. By interpolation error estimates, the efficiency and reliability of the a posteriori error estimators are proved. Finally, several numerical examples are provided to show the performance of the a posteriori error estimators.

\section{Acknowledgments}

The work of Yihui Han was supported by Guangdong Basic and Applied Basic Research Foundation (2020B1515310005) and South China Normal University Cultivation Foundation $(20 \mathrm{KJ} 18)$.

\section{References}

[1] R.A. Adams and J.J.F. Fournier. Sobolev Spaces, volume 140. Academic Press, 2003.

[2] M. Ainsworth and G. Fu. Fully computable a posteriori error bounds for hybridizable discontinuous Galerkin finite element approximations. Journal of Scientific Computing, 77(1):443-466, 2018.

[3] R. Araya, M. Solano, and P. Vega. Analysis of an adaptive HDG method for the Brinkman problem. IMA Journal of Numerical Analysis, 39(3):1502-1528, 2019.

[4] D. Boffi, F. Brezzi, and M. Fortin. Mixed Finite Element Methods and Applications, volume 44. Springer, 2013.

[5] S. Brenner and R. Scott. The Mathematical Theory of Finite Element Methods, volume 15. Springer Science \& Business Media, 2007.

[6] Z. Cai, C. He, and S. Zhang. Discontinuous finite element methods for interface problems: Robust a priori and a posteriori error estimates. SIAM Journal on Numerical Analysis, 55(1):400-418, 2017.

[7] C. Carstensen, P. Causin, and R. Sacco. A posteriori dual-mixed adaptive finite element error control for lamé and Stokes equations. Numerische Mathematik, 101(2):309-332, 2005.

[8] C. Carstensen and S. A. Funken. A posteriori error control in low-order finite element discretisations of incompressible stationary flow problems. Mathematics of Computation, 70(236):1353-1381, 2001.

[9] C. Carstensen, R. H. W. Hoppe, N. Sharma, and T. Warburton. Adaptive hybridized interior penalty discontinuous Galerkin methods for $\mathrm{H}$ (curl)-elliptic problems. Numerical Mathematics Theory Methods \& Applications, 4(001):13-37, 2011.

[10] A. Cesmelioglu, S. Rhebergen, and G. N. Wells. An embedded-hybridized discontinuous Galerkin method for the coupled Stokes-Darcy system. Journal of Computational and Applied Mathematics, 367:112476, 2020. 
[11] G. Chen, G. Fu, J. R. Singler, and Y. Zhang. A class of embedded DG methods for Dirichlet boundary control of convection diffusion PDEs. Journal of Scientific Computing, 81:623-648, 2019.

[12] H. Chen, J. Li, and W. Qiu. Robust a posteriori error estimates for HDG method for convection-diffusion equations. IMA Journal of Numerical Analysis, 36(1):437-462, 2015.

[13] H. Chen, W. Qiu, and K. Shi. A priori and computable a posteriori error estimates for an HDG method for the coercive Maxwell equations. Computer Methods in Applied Mechanics and Engineering, 333:287-310, 2018.

[14] B. Cockburn, J. Gopalakrishnan, and R. Lazarov. Unified hybridization of discontinuous Galerkin, mixed, and continuous Galerkin methods for second order elliptic problems. SIAM Journal on Numerical Analysis, 47(2):1319-1365, 2009.

[15] B. Cockburn, J. Gopalakrishnan, N.C. Nguyen, J. Peraire, and F. J. Sayas. Analysis of HDG methods for Stokes flow. Mathematics of Computation, 80(274):723-760, 2011.

[16] B. Cockburn, J. Guzmán, S.C. Soon, and H. K. Stolarski. An analysis of the embedded discontinuous Galerkin method for second-order elliptic problems. SIAM Journal on Numerical Analysis, 47(4):2686-2707, 2009.

[17] B. Cockburn and F-J. Sayas. Divergence-conforming HDG methods for Stokes flows. Mathematics of Computation, 83(288):1571-1598, 2014.

[18] B. Cockburn and W. Zhang. A posteriori error estimates for HDG methods. Journal of Scientific Computing, 51(3):582-607, 2012.

[19] B. Cockburn and W. Zhang. A posteriori error analysis for hybridizable discontinuous Galerkin methods for second order elliptic problems. SIAM Journal on Numerical Analysis, 51(1):676-693, 2013.

[20] H. Dong, B. Wang, Z. Xie, and L.L. Wang. An unfitted hybridizable discontinuous Galerkin method for the Poisson interface problem and its error analysis. IMA Journal of Numerical Analysis, 37(1):444-476, 2018.

[21] G. Fu, Y. Jin, and W. Qiu. Parameter-free superconvergent H(div)-conforming HDG methods for the Brinkman equations. IMA Journal of Numerical Analysis, (2):2, 2018.

[22] G. Fu and C-W. Shu. Analysis of an embedded discontinuous Galerkin method with implicit-explicit time-marching for convection-diffusion problems. International Journal of Numerical Analysis \& Modeling, 14(4):477-499, 2017.

[23] G. N. Gatica, L. F. Gatica, and A. Márquez. Analysis of a pseudostress-based mixed finite element method for the Brinkman model of porous media flow. Numerische Mathematik, 126(4):635-677, 2014.

[24] G. N. Gatica and F. A. Sequeira. Analysis of the HDG method for the Stokes-Darcy coupling. Numerical Methods for Partial Differential Equations, 33(3):885-917, 2017.

[25] V. Girault and P. A. Raviart. Finite Element Approximation of the Navier-Stokes Equations. Springer-Verlag, Berlin, 1979.

[26] S. Güzey, B. Cockburn, and H.K. Stolarski. The embedded discontinuous Galerkin method: application to linear shell problems. International Journal for Numerical Methods in Engineering, 70(7):757-790, 2007.

[27] Y. Han, H. Chen, X. Wang, and X. Xie. EXtended HDG methods for second order elliptic interface problems. Journal of Scientific Computing, 84(1):22, 2020.

[28] Y. Han, X. Wang, and X. Xie. An interface/boundary-unfitted eXtended HDG method for linear elasticity problems. arXiv preprint arXiv:2004.06275, 2020.

[29] A. Hannukainen, R. Stenberg, and M. Vohralík. A unified framework for a posteriori error estimation for the Stokes problem. Numerische Mathematik, 122(4):725-769, 2012. 
[30] E. Herbert and W. Christian. hp analysis of a hybrid DG method for Stokes flow. IMA Journal of Numerical Analysis, (2):687-721, 2013.

[31] P. Houston, D. Schötzau, and T. P. Wihler. Hp-adaptive discontinuous Galerkin finite element methods for the Stokes problem. In European Congress on Computational Methods in Applied Sciences and Engineering ECCOMAS, 2004.

[32] P. Houston and T. P. Schötzau, D.and Wihler. Energy norm a posteriori error estimation for mixed discontinuous Galerkin approximations of the Stokes problem. Journal of Scientific Computing, 22-23(1-3):347-370, 2005.

[33] L.N.T. Huynh, N.C. Nguyen, J. Peraire, and B.C. Khoo. A high-order hybridizable discontinuous Galerkin method for elliptic interface problems. International Journal for Numerical Methods in Engineering, 93(2):183-200, 2013.

[34] O. A. Karakashian and P. Frédéric. A posteriori error estimates for a discontinuous Galerkin approximation of second-order elliptic problems. SIAM Journal on Numerical Analysis, 41(6):2374-2399, 2003.

[35] P. L. Lederer and S. Rhebergen. A pressure-robust embedded discontinuous Galerkin method for the Stokes problem by reconstruction operators. arXiv preprint arXiv:2002.04951, 2020.

[36] H. Leng and Y. Chen. Residual-type a posteriori error analysis of HDG methods for Neumann boundary control problems. arXiv preprint arXiv:2004.09319, 2020.

[37] B. Li and X. Xie. Analysis of a family of HDG methods for second order elliptic problems. Journal of Computational and Applied Mathematics, 307:37-51, 2016.

[38] B. Li, X. Xie, and S. Zhang. Analysis of a two-level algorithm for HDG methods for diffusion problems. Communications in Computational Physics, 19(5):1435-1460, 2016.

[39] N. C. Nguyen, J. Peraire, and B. Cockburn. A class of embedded discontinuous Galerkin methods for computational fluid dynamics. Journal of Computational Physics, 302:674-692, 2015.

[40] N.C. Nguyen, J. Peraire, and B. Cockburn. A hybridizable discontinuous Galerkin method for Stokes flow. Computer Methods in Applied Mechanics and Engineering, 199(9):582-597, 2010.

[41] S. Rhebergen and G. N. Wells. Analysis of a hybridized/interface stabilized finite element method for the Stokes equations. SIAM Journal on Numerical Analysis, 55(4):1982-2003, 2017.

[42] S. Rhebergen and G. N. Wells. Preconditioning of a hybridized discontinuous Galerkin finite element method for the Stokes equations. Journal of Scientific Computing, 77(3):1936-1952, 2018.

[43] S. Rhebergen and G. N. Wells. An embedded-hybridized discontinuous Galerkin finite element method for the Stokes equations. Computer Methods in Applied Mechanics and Engineering, 358:112619, 2020.

[44] Z. Shi and M. Wang. Finite Element Methods. Science Press, 2013.

[45] R. Verfürth. A posteriori error estimators for the Stokes equations. Numerische Mathematik, 55(3):309-325, 1989.

[46] R. Verfürth. A posteriori error estimators for convection-diffusion equations. Numerische Mathematik, 80(4):641-663, 1998.

[47] B. Wang and B.C. Khoo. Hybridizable discontinuous Galerkin method (HDG) for Stokes interface flow. Journal of Computational Physics, 247(16):262-278, 2013.

[48] X. Zhang, X. Xie, and S. Zhang. An optimal embedded discontinuous Galerkin method for second-order elliptic problems. Computational Methods in Applied Mathematics, 19(4):849-861, 2019. 